\author{
Lopes, D.S., Silva, M.T., Ambrósio, J.A., Flores, P., A \\ mathematical framework for contact detection between quadric \\ and superquadric surfaces. Multibody System Dynamics, 24(3), \\ 255-280, 2010. (DOI: 10.1007/s11044-010-9220-0)
}

\title{
A MATHEMATICAL FRAMEWORK FOR RIGID CONTACT DETECTION BETWEEN QUADRIC AND SUPERQUADRIC SURFACES
}

\author{
Daniel S. Lopes ${ }^{1}$, Miguel T. Silva ${ }^{1}$, Jorge A. Ambrósio ${ }^{1}$ and Paulo Flores ${ }^{2}$ \\ ${ }^{1}$ IDMEC/Instituto Superior Técnico, Technical University of Lisbon, Av. Rovisco \\ Pais, 1049-001 Lisboa, Portugal \\ danlopes@dem.ist.utl.pt, MiguelSilva@ist.utl.pt, jorge@dem.ist.utl.pt \\ ${ }^{2}$ DEM/CT2M, Universidade do Minho, Campus Azurém, 4800-058 Guimarães, \\ Portugal \\ pflores@dem.uminho.pt
}

The calculation of the minimum distance between surfaces plays an important role in computational mechanics, namely, in the study of constrained multibody systems where contact forces take part. In this paper, a general rigid contact detection methodology for non-conformal bodies, described by ellipsoidal and superellipsoidal surfaces, is presented. The mathematical framework relies on simple algebraic and differential geometry, vector calculus, and on the $\mathrm{C}^{2}$ continuous implicit representations of the surfaces. The proposed methodology establishes a set of collinear and orthogonal constraints between vectors defining the contacting surfaces that, allied with loci constraints, which are specific to the type of surface being used, formulate the contact problem. This set of non-linear equations is solved numerically with the Newton-Raphson method with Jacobian matrices calculated analytically. The method outputs the coordinates of the pair of points with common normal vector directions and, consequently, the minimum distance between both surfaces. Contrary to other contact detection methodologies, the proposed mathematical framework does not rely on polygonal-based geometries neither on complex non-linear optimization formulations. Furthermore, the methodology is extendable to other surfaces that are (strictly) convex, interact in a non-conformal fashion, present an implicit representation, and that are at least $\mathrm{C}^{2}$ continuous. Two distinct methods for calculating the tangent and binormal vectors to the implicit surfaces are introduced: (i) a method based on the Householder reflection matrix; and (ii) a method based on a square plate rotation mechanism. The first provides a base of three orthogonal vectors, in which one of them is collinear to the surface normal. For the latter, it is 
shown that, by means of an analogy to the referred mechanism, at least two non-collinear vectors to the normal vector can be determined. Complementarily, several mathematical and computational aspects, regarding the rigid contact detection methodology, are described. The proposed methodology is applied to several case tests involving the contact between different (super)ellipsoidal contact pairs. Numerical results show that the implemented methodology is highly efficient and accurate for ellipsoids and superellipsoids.

Keywords: Minimum Distance Calculation, Rigid Contact Detection, Common Normal Concept, Superquadric Surfaces, Householder Transformation, NewtonRaphson Method.

\section{INTRODUCTION}

Contact is an omnipresent phenomenon in any mechanical system. Physically, it can be defined as the spatial configuration of two bodies on sharing a common geometric locus in which reactive forces are generated to oppose body intersection due to local deformation of the contacting surfaces and energy is dissipated, usually, in the form of heat.

In the scope of multibody dynamics [1], to accurately design and simulate a mechanism, that is either interacting with the surrounding environment or devising relative joint motion among the articulated bodies that compose it, contact forces must be utterly considered. Rigid contact analysis incorporates a computational methodology that aims to simulating the behaviour of a constrained mechanical system induced by reactive forces produced at communicating bodies. Rigid contact analysis focuses on the resolution of four fundamental issues: (i) definition of a representative geometric description of the contacting surfaces; (ii) minimum distance calculation between potential contacting surfaces; (iii) contact detection; and (iv) establishment of a constitutive force model that depends on the bodies material properties, pseudo-penetration depth and associated rate change.

One of the main requirements to model the geometry of a 3-D object, in this case the outer surface of a rigid body, is the usage of a mathematical description that provides a high geometric representativity, affiliated to a compact, controllable and intuitive set of parameters. Quadric or superquadric surfaces [2] are geometric entities that provide such a description for a variety of shapes, both natural and manmade. For mechanical systems presenting freeform shapes, superquadrics surfaces can also be applied by finding their best fit to the set of points that belong to the generalized surface $[3,4]$. This strategy is suitable to 
handle contact problems in which the contacting surfaces either have geometries close to a superquadratic surface or the surface vicinity of each contact point does not depart from a superquadric form.

The most mathematically demanding rigid contact issue concerns the minimum distance calculation. Given a non-conformal (or convex) surface representation of the colliding bodies, together with their positions and spatial orientations, the resolution of the minimum distance problem is purely geometric. The unknown variables of such problem are the spatial coordinates of the potential contacting pair of points that present collinear normal vectors. Hence, contact between two surfaces can be established by the resolution of a set of nonlinear equations that expresses collinear and orthogonal constraints between the vectors defining the contacting surfaces at the contact points, namely, the normal, tangent, binormal and distance vectors $[1,5,6,7]$. Another type of equation that is important for the geometric accuracy of contact analysis is hereafter referred as the locus (or isosurface) constraint (isoline constraint in the 2-D space). The solution of the locus constraint is the set of points that satisfy a geometric property, usually, described by an implicit surface function. As an example, the locus constraint of a sphere is the location of all the points equally distanced from its center point. Thus, for non-conformal rigid contact analysis, the calculation of the minimum distance between convex bodies requires that the surface associated with each rigid body to be described with a mathematically well-defined continuous and differentiable geometric representation, so that analytical expressions of the distance vector and the normal, tangential and binormal surface vectors can be deduced. The surface representation can be either implicit or explicit (parametric).

The intersection of rigid bodies, although physically impossible, can be considered as a motif for the mathematical concept of contact: two surfaces (or lines in the 2-D case) are in contact when their intersection is not a null set of points or, equivalently, when the lowest value of the distance function magnitude (e.g., 2-norm) is lesser or equal to zero. Rigid contact detection consists on determining if the referred bodies are sharing a common geometric locus, thus, three contact statuses are possible: (i) no contact; (ii) external contact or contact at a single point; and (iii) contact with pseudo-penetration. From a mechanical point of view, whenever contact occurs it is said that the rigid bodies overlap or present 
a pseudo-penetration. Here, contact is detected when the minimum distance is lesser than or equal to zero and positive when surfaces are apart. Note that, by convention, negative distances imply surface overlap.

The magnitude of the contact reaction forces, which depends on the minimum distance in a direct proportion [8], is calculated only when pseudopenetration occurs. Within the equations of motion, contact forces are seen as external forces that act upon interacting or interlinked bodies. Different formalisms can be used to describe contact forces, including penalty and linear complementarily contact formulations. A common model for penalty formulations was proposed by Lankarani and Nikravesh [9], based on the Hertzian non-linear elastic contact theorem [8] and on the Hunt and Crossley [10] continuous contact force model, which accounts for energy dissipation. Other formalisms, such as the linear complementarily problem [11], may also take advantage of the proposed geometric description and contact detection framework presented here, as they also need to deal with geometric modeling issues and minimum distance calculation for the evaluation of the contact forces.

Contact analysis has many important applications in other areas of applied sciences such as, molecule simulation in computational physics [12], modeling discontinuous mechanical systems (discrete element method) in geomechanics [13], humanoid design in biomechanics [14], virtual reality simulation and computer animation [15]. From such a variety of applications, several methods have been developed for distance computation and contact detection based on quadric and superquadric surfaces.

Based on an interior point algorithm, Chakraborty et al. [16] formulated the distance computation for convex bodies as an optimization problem to minimize the Euclidean distance subjected to the condition that potential contact points lie on the surfaces. Such method presents global convergence properties that are robust even in the absence of any initial information about the closest points. The method is also quite accurate since the determined points belong to the surface. Its main drawback is a lesser computational efficiency compared to other methods [13,14].

For discrete element modeling, Lin and $\mathrm{Ng}$ [13] applied contact detection algorithms for ellipsoids based on the common normal vector concept, which states that two points are the candidate contact pair of points if the normal 
directions at these points are collinear to the intersecting line. As a result, a set of non-linear equations, obtained from equaling both normal vectors and from equaling the normal vector to the distance vector, is solved numerically. Two additional conditions of the points lying on the ellipsoids were also considered. As major limitations, the method was tested only for ellipsoids and presents a system of non-linear equations that, although derived from the prescribed common normal conditions, is $\mathrm{C}^{1}$ continuous and fails when the points of contact are coincident. Such issues encourage the consideration of other relationships that include surface vectors, such as, tangents and binormals in order to rewrite the set of equations, in a more propitious form, for numerical resolution based on analytical Jacobian matrices [14].

For biomechanical applications, Kwak et al. [14] developed multibody models of diarthrodial joints, such as the knee. The models included articular contact of freeform surfaces with a parametric representation. Contact detection is also based on the common normal concept. Here, it is pointed out that the computational efficiency of their contact model is enhanced by the use of analytical Jacobians.

Wang et al. [17] presented a proximity query expressed as an algebraic condition for real-time continuous contact detection for ellipsoids moving under rigid body transformations. The algebraic condition is a quartic polynomial equation, also named as separation condition or characteristic equation, which relates the geometric parameters of shape, spatial orientation, and position of two ellipsoids. Depending on the sign of all four roots it is possible to determine the contact status. The resolution of the characteristic equation is straightforward, leading to a simple and yet efficient algorithm for contact detection of ellipsoidal bodies that computes the exact time interval of contact [18].

In this paper, a general non-conformal contact detection methodology for rigid bodies, described by ellipsoids and superellipsoids, is presented. The mathematical framework presented hereafter relies on vector calculus, algebraic and differential geometry. The proposed contact detection methodology establishes a set of geometric constraints that, once fulfilled, render the pair of points of possible contact. The resulting methodology consists in a set of nonlinear equations that is solved numerically with the Newton-Raphson method for which the Jacobian matrices are calculated analytically, without resorting to 
optimization algorithms nor polygonal-based geometries. This way, contact detection comes naturally from the minimum distance calculation. Furthermore, the methodology is extendable to other surfaces that present the following characteristics: (i) are (strictly) convex; (ii) interact in a non-conformal fashion; (iii) possess an implicit representation; (iv) and are at least $\mathrm{C}^{2}$ continuous. For the sake of simplicity, the proposed methodology is here applied only to ellipsoids and superellipsoids since these surfaces present appealing geometric properties for solution existence and uniqueness. The theoretical formulations of the geometric description and the computational implementation aspects of contact detection are described in the following sections and proper examples are provided and discussed.

\section{CONTACT DETECTION METHODOLOGY AND MULTIBODY DYNAMICS}

Detecting when and where contact occurs is of paramount importance to describe the complete dynamic response of a multibody system. The proposed contact detection methodology involves modeling the geometry of the contacting surfaces, computation of the minimum distance between surfaces and, consequently, contact detection between bodies. Within the multibody dynamics formulation, the second-order ordinary differential equations that describe the motion of a constrained mechanical system are given by [1]:

$$
\left\{\begin{array}{r}
\mathbf{M} \ddot{\mathbf{q}}+\Phi_{\mathbf{q}}^{T} \lambda=\mathbf{g} \\
\Phi_{\mathbf{q}} \ddot{\mathbf{q}}=
\end{array}\right.
$$

where $\mathbf{M}$ is the global mass matrix, $\ddot{\mathbf{q}}$ is the acceleration vector, $\boldsymbol{\Phi}_{\mathbf{q}}$ is the Jacobian matrix of the kinematic constraints, $\lambda$ is the vector of Lagrangian multipliers, $\boldsymbol{\gamma}$ is the right-hand side vector of the acceleration equation and $\mathbf{g}$ is the generalized external force vector. Spatial data, describing the position and orientation of the rigid bodies of the system, is contained within vector $\mathbf{q}$, the vector of generalized coordinates.

Contact forces are included in the $\mathbf{g}$ vector that contains all the external forces applied to the system including centrifugal and Coriolis forces. The relative 
position and orientation of the bodies, due to the application of these and other external forces, are obtained by integrating, in time, the equations of motion given by Eq. 1 in a forward dynamics perspective, as an initial value problem using the direct integration procedure [1].

Contact detection methodologies heavily rely on the evaluation of a set of geometric constraints that expresses the common normal concept $[7,13,19]$. Restriction on body movement by such constraints is imposed by the application of a pair of reactive forces that appear when surfaces overlap and not by the conditioning of a degree of freedom within the mechanical system.

For every integration time step, rigid contact detection is evaluated by testing if the bodies are apart with proximity queries $[16,18]$ or, when these fail, by determining the minimum Euclidean distance between the surfaces. When two moving surfaces overlap, a pair of opposite contact forces appear, each one applied on the contact points of the intervening bodies and with the direction of the minimum distance vector. The reaction force magnitude is proportional to the pseudo-penetration. Although the contact detection methodology regards bodies as being rigid, the pseudo-penetration is an estimate of the deformation that results from the surface overlap. This way, not only contact forces can be predicted but also contact areas and contact stresses can be estimated [14].

In Fig. 1, a generalized multibody model of a contact pair of bodies is presented. Each rigid body is defined with at least three points using a multibody formulation with natural coordinates [20] and can contain more than one contact surface. The coordinate systems employed to describe the motion and configuration of a rigid contact pair are: the global coordinate system $X Y Z$; the fixed coordinate systems $\xi_{k} \eta_{k} \zeta_{k}$ of rigid bodies $k, k \in\{\alpha, \beta\}$; and the local coordinate systems $x_{m} y_{m} z_{m}$ of surfaces $m, m \in\{i, j\}$. Position vectors and rotation matrices, obtained from the multibody dynamics calculations are represented, respectively, by $\mathbf{r}$ and $\mathbf{A}$ where the subscripts indicate the corresponding coordinates systems, e.g., $\mathbf{A}_{\mathrm{O} \beta}$ represents the coordinate transformation from the fixed coordinate system of body $\beta$ to the global coordinate system. Position vectors in the local reference system of the contacting surface are denoted by $\mathbf{s}$. Each body has a fixed coordinate system that must not be confused with the local coordinate systems of the surfaces that compose it. Note that, all vector relationships that define the geometric constraints are referenced to the global 
coordinate system and, since the methodology is designed for non-conformal contact, i.e., interacting surfaces have dissimilar profiles, contact calculation is performed using the outward surface normals.

Figure 1: Multibody model of a contact pair and vector entities involved in contact analysis along with the coordinates systems (global, rigid body and surface reference frames). The detail shows the intervening vector quantities of proposed contact detection formulation.

\section{MATHEMATICAL FORMULATION FOR MINIMUM DISTANCE CALCULATION AND CONTACT DETECTION}

The minimum distance calculation between two rigid, non-conformal, and (strictly) convex surfaces can be formulated as a constraint optimization problem in which the objective function is defined by the 2-norm distance between surface points and where the constraints are derived from the common normal concept $[16,21]$. From non-linear optimization, it is well known that the minimum distance calculation is a problem for which the existence of a minimizing solution is proven by Weierstrass' theorem and whose unicity of solution is proven by second-order optimality conditions [22]. The minimum distance calculation problem is also well-posed for the surface types under consideration since the solution depends continuously on the data.

Rather than dealing with complex and time consuming optimization schemes $[16,21,23,24]$, the constraint optimization problem can be transformed into a system of non-linear equations [23] where the minimum distance problem is written, solely, as the set of the geometric constraints that express the common normal conditions, thus, no non-linear objective function is required. With the problem written as a system of non-linear equations, it is possible to explore the potential of the Newton-Raphson's method efficiency since it presents a quadratic convergence behaviour; an important feature when regarding real-time contact detection. But in order to implement an efficient Newton-Raphson method it is necessary to surpass the method's major numerical restrictions [23,25]: (i) the analytical Jacobian matrix is needed, therefore, analytical derivatives must be available [14]; (ii) function evaluation must be inexpensive; and (iii) since it is a 
local convergence method, the success of the algorithm deeply depends on the initial approximation.

The following sub-sections present a formulation of the minimum distance calculation problem, expressed as a system of non-linear equations, which is generic to $\mathrm{C}^{2}$ continuous implicit surfaces along with a contact detection formulation that is particularized to radially symmetric, convex and closed surfaces, such as, (super)ellipsoids.

\subsection{Problem statement}

Consider two non-deformable bodies $\alpha$ and $\beta$ whose global positions and orientations are given. Rigidly coupled to each body are two non-conformal $\mathrm{C}^{2}$ continuous surfaces $i$ and $j$ that bear implicit and/or parametric representations. The minimum distance problem consists of determining the surface points that share a common normal and where the distance between surfaces is minimal. The common normal concept states the necessary conditions for that two points form a contact pair: the normal vectors at these points must be collinear relatively to each other and collinear with the distance vector that connects the two points (Fig. 1). From this enunciation, it can be inferred that the mathematical formulation for contact is intimately related to the surface representation. Implicit and parametric surface representations are written, in a general form, as:

$$
\begin{aligned}
& \mathcal{F}_{i}\left(x_{i}, y_{i}, z_{i}\right)=0, \\
& \mathcal{F}_{j}\left(x_{j}, y_{j}, z_{j}\right)=0,
\end{aligned}
$$

and

$$
\begin{aligned}
\mathbf{p}_{i} & =\mathbf{p}_{i}(s, u), \\
\mathbf{p}_{j} & =\mathbf{p}_{j}(w, v),
\end{aligned}
$$

where $\mathcal{F}_{i}$ and $\mathcal{F}_{j}$ are implicit scalar functions that define the locus of points belonging to surfaces $i$ and $j$, respectively, $\mathbf{p}_{i}$ and $\mathbf{p}_{j}$ are the parametric position vectors of the referred surfaces, $(x, y, z)$ the Cartesian coordinates of a surface point and $(s, u)$ and $(w, v)$ their respective parametric coordinates. The distance vector, $\mathbf{d}_{\mathrm{PQ}}$, that connects the two minimum distance surface points, $\mathrm{P}$ and $\mathrm{Q}$, is defined as the difference between the position vectors of points $\mathrm{P}$ and $\mathrm{Q}$, given by $\mathbf{r}_{\mathrm{OP}}$ and $\mathbf{r}_{\mathrm{OQ}}$, as represented in Fig. 1. Thus, the distance vector is written as: 


$$
\mathbf{d}_{\mathrm{PQ}}=\mathbf{r}_{\mathrm{OQ}}-\mathbf{r}_{\mathrm{OP}}
$$

It should be noted that $\mathbf{r}_{\mathrm{OP}}$ and $\mathbf{r}_{\mathrm{OQ}}$ are unknown quantities, i.e., the coordinates of these points are the aim of minimum distance calculations. The normal vector at each point, $\mathbf{n}_{\mathrm{OP}}$ and $\mathbf{n}_{\mathrm{OQ}}$, is derived by partial differentiation of the surface equation in order to the spatial coordinates. If the implicit surface representation, given in Eq. 2, is considered then the normal vector at a given point is the surface gradient evaluated at that point. The tangent and binormal vectors at each point, $\mathbf{t}_{\mathrm{OP}}, \mathbf{t}_{\mathrm{OQ}}, \mathbf{b}_{\mathrm{OP}}$ and $\mathbf{b}_{\mathrm{OQ}}$, are contained within the plane defined by the respective normal vectors and candidate points. In the case of parametric surfaces, defined according to Eq. 3, the differentiation of the surface expression gives the tangential vectors along each spatial parameter and, in this case, the surface normals are calculated by the cross product of the tangent vectors. For implicit surfaces, the tangent and binormal vectors must be obtained in a more contriving manner as it will be seen later in section 3.4.

\subsection{Geometric constraints}

In this work, rigid contact detection is formulated as a set of geometric constraints that are used to describe the referred common normal concept. These constrained equations are written as a vector $\boldsymbol{\Phi}^{G}$ that depends on the vector of the unknown points coordinates, $\mathbf{q}^{G}$,

$$
\boldsymbol{\Phi}^{G}=\boldsymbol{\Phi}^{G}\left(\mathbf{q}^{G}\right)
$$

where the superscript ' $G$ ' is used to classify the geometric constraint equations within the multibody formulation.

In general, three types of non-linear equations are used to define a set of geometric constraints for minimum distance calculation: (i) orthogonal constraint (relative constraint between two perpendicular vectors); (ii) collinear constraint (relative constraint between two parallel vectors); and (iii) locus or isosurface constraint (assures that a point in space lies on a contact surface). It should be noticed that the latter type of constraint is only introduced for contact detection regarding implicit surfaces. Moreover, for convenience of the methodology, vector $\mathbf{q}^{G}$ is expressed with respect to the local reference frame attached to the intervening surfaces, as it will be described in the next section. 
As it was mentioned previously, minimum distance calculation between surfaces is stated as the geometric problem consisting of encountering the location of the pair of points $\mathrm{P}$ and $\mathrm{Q}$ in which the distance vector, $\mathbf{d}_{\mathrm{PQ}}$, is aligned to the surface normals $\mathbf{n}_{\mathrm{OP}}$ and $\mathbf{n}_{\mathrm{OQ}}$, and has a minimum 2-norm value. The collinearity condition between these vectors can be written as two cross product equations relating vectors $\mathbf{d}_{\mathrm{PQ}}, \mathbf{n}_{\mathrm{OP}}$, and $\mathbf{n}_{\mathrm{OQ}}$. Equivalently, this can also be expressed as an orthogonal constraint involving vector $\mathbf{d}_{\mathrm{PQ}}$, surface tangent vectors $\mathbf{t}_{\mathrm{OP}}$ and $\mathbf{t}_{\mathrm{OQ}}$, and binormal vectors $\mathbf{b}_{\mathrm{OP}}$ and $\mathbf{b}_{\mathrm{OQ}}$. Equation 6a presents this equivalency:

$$
\begin{gathered}
\mathbf{d}_{\mathrm{PQ}} \times \mathbf{n}_{\mathrm{OP}}=\mathbf{0} \Leftrightarrow\left\{\begin{array}{l}
\mathbf{d}_{\mathrm{PQ}} \cdot \mathbf{t}_{\mathrm{OP}}=0 \\
\mathbf{d}_{\mathrm{PQ}} \cdot \mathbf{b}_{\mathrm{OP}}=0
\end{array}\right. \\
\mathbf{d}_{\mathrm{PQ}} \times \mathbf{n}_{\mathrm{OQ}}=\mathbf{0} \Leftrightarrow\left\{\begin{array}{l}
\mathbf{d}_{\mathrm{PQ}} \cdot \mathbf{t}_{\mathrm{OQ}}=0 \\
\mathbf{d}_{\mathrm{PQ}} \cdot \mathbf{b}_{\mathrm{OQ}}=0
\end{array}\right.
\end{gathered}
$$

Other collinearity condition that is verified at the minimum distance points is:

$$
\begin{aligned}
& \mathbf{n}_{\mathrm{OP}} \times \mathbf{n}_{\mathrm{OQ}}=\mathbf{0} \Leftrightarrow\left\{\begin{array}{l}
\mathbf{n}_{\mathrm{OP}} \cdot \mathbf{t}_{\mathrm{OQ}}=0 \\
\mathbf{n}_{\mathrm{OP}} \cdot \mathbf{b}_{\mathrm{OQ}}=0
\end{array}\right. \\
& \mathbf{n}_{\mathrm{OQ}} \times \mathbf{n}_{\mathrm{OP}}=\mathbf{0} \Leftrightarrow\left\{\begin{array}{l}
\mathbf{n}_{\mathrm{OQ}} \cdot \mathbf{t}_{\mathrm{OP}}=0 \\
\mathbf{n}_{\mathrm{OQ}} \cdot \mathbf{b}_{\mathrm{OP}}=0
\end{array}\right.
\end{aligned}
$$

Such a condition (Eq. 6b) is equivalent to the orthogonality constraints between the normal vector of one of the surfaces relatively to the tangent and binormal vectors of the paired surface. Equations 2, Eq. 6a and Eq. $6 \mathrm{~b}$ utterly define the common normal concept.

In 3-D space, whether one considers the collinear or orthogonal constraint definition, Eq. 6a and Eq. 6b always render a system of four non-linear independent equations. In the case of the parametric surface representation, Eq. 6a and Eq. $6 \mathrm{~b}$ can be solved for the 4 unknown surface parameters $(s, u, w, v)$. Alternatively, when the surface representation follows an implicit definition, 2 additional locus constraints, given by Eq. 2, must be added, one for each rigid surface, to the set of orthogonal constraints, providing the $4+2$ equations necessary for solving the 6 Cartesian coordinates $\left(x_{i}, y_{i}, z_{i}\right)$ and $\left(x_{j}, y_{j}, z_{j}\right)$ of the two potential contact points. Redundant constraints must be avoided so that all the equations are linearly independent. The geometric constraint equations (Eq. 2, Eq. 6a, Eq. 6b) serve to restrict the solution space of $\boldsymbol{\Phi}^{G}$ to the solution with most physical meaning for contact. 


\subsection{Vector of geometric constraints and analytical Jacobian matrix}

When dealing with $C^{2}$ surfaces, such as (super)ellipsoidal surfaces, the vector of geometric constraints $\boldsymbol{\Phi}^{G}$ is built with $\mathrm{C}^{2}$ continuous functions and the dot and cross product operators do not introduce any discontinuity in the domain, therefore, $\boldsymbol{\Phi}^{G}$ is a twice-differentiable vector function. The system of non-linear equations that contains these non-redundant geometric constraints,

$$
\boldsymbol{\Phi}^{G}\left(\mathbf{q}^{G}\right)=\mathbf{0},
$$

can then be solved using the Newton-Raphson iterative procedure. Since $\boldsymbol{\Phi}^{G}$ has, at least, a $\mathrm{C}^{2}$ mathematical expression, one can exploit the numerical behaviour with analytical Jacobians. Hence:

$$
\mathbf{q}_{k+1}^{G}=\mathbf{q}_{k}^{G}-\left(\boldsymbol{\Phi}_{\mathbf{q}^{G}}^{G}\left(\mathbf{q}_{k}^{G}\right)\right)^{-1} \boldsymbol{\Phi}^{G}\left(\mathbf{q}_{k}^{G}\right),
$$

where $\boldsymbol{\Phi}_{\mathbf{q}}{ }^{G}$ is the Jacobian matrix of the geometric constraints and $k$ the NewtonRaphson iterator index. The Newton-Raphson scheme iterates until the difference between $\mathbf{q}_{k+1}^{G}$ and $\mathbf{q}_{k}^{G}$ is lesser than a user specified tolerance. A rapid convergence is usually obtained considering good initial guesses for $\mathbf{q}_{0}^{G}$.

There is more than one way to formulate distance computation via systems of geometric constraint equations (Eq. 2, Eq. 6a, Eq. 6b) as the formulation deeply depends on the analytical behaviour of the functions at stake and on the surface representation type, e.g., when considering parametric surfaces loci constraints are, obviously, not relevant and only orthogonal or collinear constraints are of interest. In this work, mathematical modeling for contact detection exploits geometric properties associated with the implicit surface representation of (super)ellipsoids in order to define the mathematical entities (e.g., vectors and surface functions) that participate in the geometric constraint equations. Additionally, other geometric properties, such as the radial symmetry, are useful to guarantee unicity of the minimum distance problem as described in section 3.5.

From the set of geometric conditions that are verified at the points of minimum distance (Eq. 2, Eq. 6a, Eq. 6b), i.e., 4 collinear constraints, 8 orthogonal constraints and 2 loci constraints, it is necessary to elect 6 equations, two of them are, obligatorily, the locus constraints of each implicit surface and 
the remaining 4 are selected such that no linear dependent equations are inserted in vector $\boldsymbol{\Phi}^{G}$.

For 3-D (strictly) convex and closed objects represented implicitly (Fig. 1), the abovementioned geometric constraints of orthogonality and isosurface are grouped in a 6x1 vector $\boldsymbol{\Phi}^{(G, 6)}$ which can be written in the homogeneous form as,

$$
\boldsymbol{\Phi}^{(G, 6)}\left(\mathbf{q}^{G}\right)=\mathbf{0} \Leftrightarrow\left[\begin{array}{c}
\mathbf{n}_{\mathrm{OP}}^{T} \cdot \mathbf{t}_{\mathrm{OQ}} \\
\mathbf{n}_{\mathrm{OP}}^{T} \cdot \mathbf{b}_{\mathrm{OQ}} \\
\mathbf{d}_{\mathrm{PQ}}^{T} \cdot \mathbf{t}_{\mathrm{OQ}} \\
\mathbf{d}_{\mathrm{PQ}}^{T} \cdot \mathbf{b}_{\mathrm{OQ}} \\
\mathcal{F}_{i} \\
\mathcal{F}_{j}
\end{array}\right]=\left[\begin{array}{l}
0 \\
0 \\
0 \\
0 \\
0 \\
0
\end{array}\right],
$$

where

$$
\mathbf{q}^{G}=\left[x_{i}, y_{i}, z_{i}, x_{j}, y_{j}, z_{j}\right]^{T}=\left[\begin{array}{ll}
\mathbf{s}_{i \mathrm{P}}^{T} & \mathbf{s}^{T}{ }_{\mathrm{i}}
\end{array}\right]^{T},
$$

is the vector that contains the coordinates of the potential contact pair of points expressed in the local reference frame associated with the implicit surface representation.

The last two geometric constraints in Eq. 9 are the implicit surface equations given in the canonical form, i.e., the surface principal axes are aligned with the local coordinate axes and the surface centroid is coincident with the local origin, as represented in the two ellipsoidal surfaces depicted in Fig. 1.

All vectors in Eq. 9 are defined in the global coordinate system. However, since $\mathbf{q}^{G}$ holds the coordinates of the possible contact pair of points in the local coordinate system of the intervening surfaces, proper coordinate transformations need to be introduced to express these vectors as a function of $\mathbf{q}^{G}$. Resorting to Fig. 1, the following expressions are used to calculate the global coordinates of vectors $\mathbf{d}_{\mathrm{PQ}}, \mathbf{n}_{\mathrm{OP}}, \mathbf{t}_{\mathrm{OQ}}$ and $\mathbf{b}_{\mathrm{OQ}}$ :

$$
\begin{aligned}
\mathbf{n}_{\mathrm{OP}} & =\mathbf{A}_{\mathrm{O} \alpha} \mathbf{A}_{\alpha i} \mathbf{n}_{i \mathrm{P}}, \\
\mathbf{t}_{\mathrm{OQ}} & =\mathbf{A}_{\mathrm{O} \beta} \mathbf{A}_{\beta j} \mathbf{t}_{j \mathrm{Q}}, \\
\mathbf{b}_{\mathrm{OQ}} & =\mathbf{A}_{\mathrm{O} \beta} \mathbf{A}_{\beta j} \mathbf{b}_{j \mathrm{Q}}, \\
\mathbf{d}_{\mathrm{PQ}} & =\mathbf{r}_{\mathrm{OQ}}-\mathbf{r}_{\mathrm{OP}}= \\
& =\mathbf{r}_{\mathrm{O} \beta}+\mathbf{A}_{\mathrm{O} \beta} \mathbf{r}_{\beta j}+\mathbf{A}_{\mathrm{O} \beta} \mathbf{A}_{\beta j} \mathbf{s}_{j \mathrm{Q}}-\left(\mathbf{r}_{\mathrm{O} \alpha}+\mathbf{A}_{\mathrm{O} \alpha} \mathbf{r}_{\alpha i}+\mathbf{A}_{\mathrm{O} \alpha} \mathbf{A}_{\alpha i} \mathbf{s}_{i \mathrm{P}}\right),
\end{aligned}
$$


where $\mathbf{n}_{i \mathrm{P}}, \mathbf{t}_{j \mathrm{Q}}$, and $\mathbf{b}_{j \mathrm{Q}}$ are the local coordinates of these vectors expressed in the surface reference frame, $\mathbf{A}_{\mathrm{O} \alpha}, \mathbf{A}_{\mathrm{O} \beta}, \mathbf{A}_{\alpha i}$, and $\mathbf{A}_{\beta j}$ are the rotation matrices, $\mathbf{r}_{\mathrm{O} \alpha}, \mathbf{r}_{\mathrm{O} \beta}$, $\mathbf{r}_{\alpha i}$, and $\mathbf{r}_{\beta j}$ are the origin positions of the rigid body and surface reference frames, and $\mathbf{s}_{i \mathrm{P}}$ and $\mathbf{s}_{j \mathrm{Q}}$ are the local position vectors of points $\mathrm{P}$ and $\mathrm{Q}$ as referred in Section 2.

The Newton-Raphson method relies on the Taylor series $1^{\text {st }}$ order expansion of $\boldsymbol{\Phi}^{(G, 6)}$. This demands the calculation of the $6 \times 6$ Jacobian matrix of the geometric constraints. By definition, each row of the Jacobian matrix is the first partial derivative of $\boldsymbol{\Phi}^{(G, 6)}$ in order to $\mathbf{q}^{G}$. Hence:

$$
\boldsymbol{\Phi}_{\mathbf{q}^{G}}^{G}\left(\mathbf{q}^{G}\right)=\frac{\partial}{\partial \mathbf{q}^{G}} \boldsymbol{\Phi}^{(G, 6)}\left(\mathbf{q}^{G}\right) \Leftrightarrow \boldsymbol{\Phi}_{\mathbf{q}^{G}}^{G}\left(\mathbf{q}^{G}\right)=\left[\begin{array}{c}
\mathbf{t}_{\mathrm{OQ}}^{T}\left(\mathbf{n}_{\mathrm{OP}}\right)_{\mathbf{q}^{G}}+\mathbf{n}_{\mathrm{OP}}^{T}\left(\mathbf{t}_{\mathrm{OQ}}\right)_{\mathbf{q}^{G}} \\
\mathbf{b}_{\mathrm{OQ}}^{T}\left(\mathbf{n}_{\mathrm{OP}}\right)_{\mathbf{q}^{G}}+\mathbf{n}_{\mathrm{OP}}^{T}\left(\mathbf{b}_{\mathrm{OQ}}\right)_{\mathbf{q}^{G}} \\
\mathbf{t}_{\mathrm{OQ}}^{T}\left(\mathbf{d}_{\mathrm{PQ}}\right)_{\mathbf{q}^{G}}+\mathbf{d}_{\mathrm{PQ}}^{T}\left(\mathbf{t}_{\mathrm{OQ}}\right)_{\mathbf{q}^{G}} \\
\mathbf{b}_{\mathrm{OQ}}^{T}\left(\mathbf{d}_{\mathrm{PQ}}\right)_{\mathbf{q}^{G}}+\mathbf{d}_{\mathrm{PQ}}^{T}\left(\mathbf{b}_{\mathrm{OQ}}\right)_{\mathbf{q}^{G}} \\
\mathbf{n}_{i \mathrm{P}}^{T} \\
\mathbf{0}_{1 \times 3} \\
\mathbf{n}_{j \mathrm{Q}}^{T}
\end{array}\right]
$$

where, considering Eq. 11,

$$
\begin{aligned}
\left(\mathbf{n}_{\mathrm{OP}}\right)_{\mathbf{q}^{G}} & =\mathbf{A}_{\mathrm{O} \alpha} \mathbf{A}_{\alpha i}\left(\mathbf{n}_{i \mathrm{P}}\right)_{\mathbf{q}^{G}}, \\
\left(\mathbf{t}_{\mathrm{OQ}}\right)_{\mathbf{q}^{G}} & =\mathbf{A}_{\mathrm{O} \beta} \mathbf{A}_{\beta j}\left(\mathbf{t}_{j \mathrm{Q}}\right)_{\mathbf{q}^{G}}, \\
\left(\mathbf{b}_{\mathrm{OQ}}\right)_{\mathbf{q}^{G}} & =\mathbf{A}_{\mathrm{O} \beta} \mathbf{A}_{\beta j}\left(\mathbf{b}_{j \mathrm{Q}}\right)_{\mathbf{q}^{G}}, \\
\left(\mathbf{d}_{\mathrm{PQ}}\right)_{\mathbf{q}^{G}} & =\mathbf{A}_{\mathrm{O} \beta} \mathbf{A}_{\beta j}\left(\mathbf{s}_{j \mathrm{Q}}\right)_{\mathbf{q}^{G}}-\mathbf{A}_{\mathrm{O} \alpha} \mathbf{A}_{\alpha i}\left(\mathbf{s}_{i \mathrm{P}}\right)_{\mathbf{q}^{G}},
\end{aligned}
$$

are $3 \times 6$ matrices and

$$
\left(\mathbf{s}_{i \mathbf{P}}\right)_{\mathbf{q}^{G}}=\left[\begin{array}{lll:lll}
1 & 0 & 0 & 0 & 0 & 0 \\
0 & 1 & 0 & 0 & 0 & 0 \\
0 & 0 & 1 & 0 & 0 & 0
\end{array}\right]=\left[\begin{array}{l}
\mathbf{I} \\
\mathbf{0}
\end{array}\right], \quad\left(\mathbf{s}_{j \mathrm{Q}}\right)_{\mathbf{q}^{G}}=\left[\begin{array}{lll:lll}
0 & 0 & 0 & 1 & 0 & 0 \\
0 & 0 & 0 & 0 & 1 & 0 \\
0 & 0 & 0 & 0 & 0 & 1
\end{array}\right]=\left[\begin{array}{ll}
\mathbf{0} & \mathbf{I}
\end{array}\right] .
$$

The equations presented in this section are generic to any implicit surface that is $\mathrm{C}^{2}$ continuous and, once equations Eq. 9 and Eq. 12 are implemented, extending the methodology to other surfaces requires the deduction of the analytical expressions for $\mathbf{n}_{i \mathrm{P}}, \mathbf{t}_{j \mathrm{Q}}, \mathbf{b}_{j \mathrm{Q}},\left(\mathbf{n}_{i \mathrm{P}}\right)_{\mathbf{q}^{G}},\left(\mathbf{t}_{j \mathrm{Q}}\right)_{\mathbf{q}^{G}}$, and $\left(\mathbf{b}_{j \mathrm{Q}}\right)_{\mathbf{q}^{G}}$ which is, usually, a straightforward process considering the analytical nature of the surfaces definitions. Despite the generic nature of the method, the geometric constraints 
presented here are only necessary conditions for contact detection, although in some cases, such as two non-aligned ellipsoids, the conditions reveal to be sufficient. Therefore, extending the formulation to other implicit surfaces entails additional considerations that must guarantee the existence and unicity of the minimum distance solution. For example, in the case of open surfaces, such as the paraboloid, the boundaries are required to be limited and the geometric equations that define the boundaries must enter the vector of geometric constraints, in order to evaluate if the potential contact points are contained within the surface boundaries.

\subsection{Tangent and binormal vectors given an arbitrary normal vector}

When dealing with $\mathrm{C}^{n}, n \geq 1$, implicit surfaces, it is relatively easy to obtain the normal vector at any point on the surface. The gradient is a differential operator that once applied to the analytical surface expression (Eq. 2) defines the normal vector at each point. However, the tangent and binormal vectors are not calculated just as simply. Although it seems intuitive, finding orthogonal vectors given an arbitrary non null vector in 3-D Cartesian space is not a trivial task. Here, two different approaches for calculating an orthogonal vector basis, that belongs to the tangent plane defined by the gradient normal vector, are considered: (i) by determining an appropriate Householder reflection matrix; and (ii) by the cross product of the normal vector with an auxiliary non-collinear vector. Both approaches lead to analytical expressions for the tangent and binormal vectors which depend on the coordinates of the normal vector and, consequently, on the local surface point coordinates.

The Householder transformation [26] is expressed as a matrix $\mathbf{H}$ which reflects a vector, in this case the normal vector $\mathbf{n}$, along a vector axis $\mathbf{h}$ :

$$
\mathbf{H}=\mathbf{I}-2 \frac{\mathbf{h h}^{T}}{\mathbf{h}^{T} \mathbf{h}}=\left[\begin{array}{ccc}
1-2 \frac{h_{1}^{2}}{h^{2}} & -2 \frac{h_{1} h_{2}}{h^{2}} & -2 \frac{h_{1} h_{3}}{h^{2}} \\
-2 \frac{h_{1} h_{2}}{h^{2}} & 1-2 \frac{h_{2}^{2}}{h^{2}} & -2 \frac{h_{2} h_{3}}{h^{2}} \\
-2 \frac{h_{1} h_{3}}{h^{2}} & -2 \frac{h_{2} h_{3}}{h^{2}} & 1-2 \frac{h_{3}^{2}}{h^{2}}
\end{array}\right],
$$

where 


$$
\mathbf{h} \equiv \mathbf{h}(\mathbf{n})=\left[\begin{array}{lll}
h_{1} & h_{2} & h_{3}
\end{array}\right]^{T}=\left[\begin{array}{lll}
n_{x}+\|\mathbf{n}\|_{2} & n_{y} & n_{z}
\end{array}\right]^{T}
$$

and

$$
h=\|\mathbf{h}\|_{2} .
$$

Matrix $\mathbf{H}$ is symmetric and orthogonal with columns (or rows) forming an orthogonal vector basis. The first column of $\mathbf{H}$ is collinear to $\mathbf{n}$ and the remaining columns are perpendicular to $\mathbf{n}$. Here, $\mathbf{t} \equiv \mathbf{t}(\mathbf{n})$ and $\mathbf{b} \equiv \mathbf{b}(\mathbf{n})$ are assigned as the second and third columns, respectively. Towards the methodology, Eq. 15 acts, merely, as a formula to calculate the tangent and binormal vectors at a given surface point.

Inspired by the motion of a simple rotation mechanism (Fig. 2), an alternative way to determine tangent and binormal vectors is to define a set of four vectors in which at least two of them are non-collinear to an arbitrary vector $\mathbf{n}$ (Fig. 3):

$$
\mathbf{v}_{1}=\left[\begin{array}{c}
n_{x}+\|\mathbf{n}\|_{2} \\
n_{y}+\|\mathbf{n}\|_{2} \\
n_{z}
\end{array}\right], \quad \mathbf{v}_{2}=\left[\begin{array}{c}
n_{x}-\|\mathbf{n}\|_{2} \\
n_{y}+\|\mathbf{n}\|_{2} \\
n_{z}
\end{array}\right], \quad \mathbf{v}_{3}=\left[\begin{array}{c}
n_{x}-\|\mathbf{n}\|_{2} \\
n_{y}-\|\mathbf{n}\|_{2} \\
n_{z}
\end{array}\right], \quad \mathbf{v}_{4}=\left[\begin{array}{c}
n_{x}+\|\mathbf{n}\|_{2} \\
n_{y}-\|\mathbf{n}\|_{2} \\
n_{z}
\end{array}\right]
$$

Figure 2: Square plate rotation mechanism. The revolute joints rotate at the same angular velocity but in opposite senses.

In general, all four vectors in Eq. 18 are not collinear to $\mathbf{n}$, except when $n_{x}=n_{y}$ or $n_{x}=-n_{y}$, in which case, only two non-collinear vectors are available (see rightmost graph of Fig. 3). Since only one non-collinear vector is required to obtain the tangent and binormal, the selection of the non-collinear vector is, here, undertook as listed in Table 1.

Figure 3: "Proof by picture" of the existence of non-collinear vectors given an arbitrary vector $\mathbf{n}_{x y}$ in the 2-D plane.

Despite the fact that the proof is referred to the 2-D plane, i.e., $n_{z}=0$ (Fig. 3 ), the proof is clearly valid for any $3-\mathrm{D}$ vector as $n_{z}$ can take real values different from zero, $n_{z} \neq 0$. 
Applying the cross product operator between the normal and the elected non-collinear vector $\mathbf{v}_{l}, l \in\{1,2,3,4\}$, the tangent and binormal vectors can then be calculated with Eq. 19 .

$$
\begin{aligned}
& \mathbf{t} \equiv \mathbf{t}(\mathbf{n})=\mathbf{n} \times \mathbf{v}_{l} \\
& \mathbf{b} \equiv \mathbf{b}(\mathbf{n})=\mathbf{n} \times \mathbf{t}=\mathbf{n} \times\left(\mathbf{n} \times \mathbf{v}_{l}\right)
\end{aligned}
$$

According to the formalism of Eq. 13, the Jacobian matrices are expressed as:

$$
\begin{aligned}
& \left(\mathbf{t}_{\mathrm{OQ}}\right)_{\mathbf{q}^{G}}=\mathbf{A}_{\mathrm{O} \beta} \mathbf{A}_{\beta j}\left[\mathrm{~A} / g_{\mathrm{Q}}\left(\mathbf{v}_{j \mathrm{Q}}\right)_{\mathbf{q}^{G}}+\mathscr{Q} / g_{\mathrm{Q}}\left(\mathbf{n}_{j \mathrm{Q}}\right)_{\mathbf{q}^{G}}\right],
\end{aligned}
$$

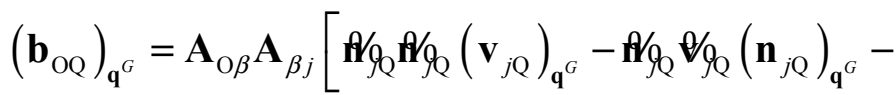

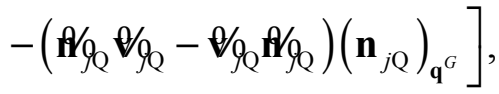

where the tilde sign $(\sim)$ above the vector notation is the skew-symmetric matrix associated with the corresponding vector.

Table 1: Selected pairs of non-collinear vectors according to the signs of the $n_{x}$ and $n_{y}$ coordinates.

\subsection{Contact detection}

The distance vector magnitude, $d$, is calculated as the signed Euclidean distance of vector $\mathbf{d}_{\mathrm{PQ}}$, thus, 'negative distances' are considered. At a given time instant, the signed magnitude $d$ indicates one of the three possible contact situations as summarized in Table 2: (i) no contact; (ii) contact at a single point or external contact $\left(\mathbf{r}_{\mathrm{OP}}=\mathbf{r}_{\mathrm{OQ}}\right)$ and (iii) contact with pseudo-penetration. Therefore, by computing the minimum distance between rigid surfaces it is possible to detect the contact state.

Table 2: Contact detection situations according to the minimum distance value.

Note that the formulated vector of geometric constraints, $\boldsymbol{\Phi}^{G}$ (Eq. 9), reckons only the common normal concept, meaning that, for some contact pairs, it does not formulate the minimum distance calculation per se. Consequently, multiple pairs of points with common normals that are collinear with the distance vector may appear. In the case of (super)ellipsoids (Fig. 4), 2 or 4 multiple 
solutions of $\boldsymbol{\Phi}^{G}$ may result if one of the following situations occurs: (i) whenever spheres take part of the contact pair ( 2 or 4 solutions); (ii) whenever both surfaces are aligned, i.e., when two of the surface's planes of symmetry are coincident (4 solutions).

Figure 4: Multiple solutions for the common normal concept. A1 - Contact pair formed by a sphere and an ellipsoid that are aligned with each other; A2 - Multiple solutions of the corresponding vector of geometric constraints $\boldsymbol{\Phi}^{G}$; B1 - Contact pair formed by a sphere and an ellipsoid that are not aligned with each other; B2 - Multiple solutions of the corresponding vector of geometric constraints $\boldsymbol{\Phi}^{G}$.

In order to guarantee the determination of the proper solution to the iterative procedure, one must grant an approximated initial position that is close to the desired solution. This is also a requirement for the convergence of the Newton-Raphson method. Even if the numerical method does not converge to the minimum result, it is convenient to make use of the (super)ellipsoids geometric properties and intrinsic characteristics, such as, convexity, compactness (closed and bounded surface) and radial symmetry, to ascertain the unique minimum distance solution.

Thus, once the method converges, and after converting $\mathbf{q}_{k_{\text {final }}}^{G}$ to global coordinates, it is necessary to inquire if the obtained result,

$$
\mathbf{q}^{*}=\left[\begin{array}{l}
\mathbf{q}_{1}^{*} \\
\mathbf{q}_{2}^{*}
\end{array}\right]
$$

corresponds to the minimum distance solution. Given two arbitrary (super)ellipsoids in a non-conformal contact situation, there are, at most, a total of four possible solutions of $\boldsymbol{\Phi}^{G}$ :

$$
\mathrm{K}=\left\{\left[\begin{array}{c}
\mathbf{q}_{1}^{*} \\
\mathbf{q}_{2}^{*}
\end{array}\right],\left[\begin{array}{c}
\mathbf{q}_{1}^{*} \\
\mathbf{q}_{4}^{*}
\end{array}\right],\left[\begin{array}{c}
\mathbf{q}_{3}^{*} \\
\mathbf{q}_{2}^{*}
\end{array}\right],\left[\begin{array}{c}
\mathbf{q}_{3}^{*} \\
\mathbf{q}_{4}^{*}
\end{array}\right]\right\} \quad \text { with } \quad \mathbf{q}_{3}^{*}=-\mathbf{q}_{1}^{*} \text { and } \mathbf{q}_{4}^{*}=-\mathbf{q}_{2}^{*} \text {, }
$$

where the points $\mathbf{q}_{1}^{*}$ and $\mathbf{q}_{3}^{*}$ belong to surface $i$ and $\mathbf{q}_{2}^{*}$ and $\mathbf{q}_{4}^{*}$ belong to surface $j$. Hence, the position vectors of the minimum distance points $\mathrm{P}$ and $\mathrm{Q}$ are such that:

$$
\mathbf{r}_{\mathrm{OP}} \in\left\{\mathbf{q}_{1}^{*}, \mathbf{q}_{3}^{*}\right\} \text { and } \mathbf{r}_{\mathrm{OQ}} \in\left\{\mathbf{q}_{2}^{*}, \mathbf{q}_{4}^{*}\right\} \text {. }
$$


By evaluating the Euclidean distance between all four combinations of the subvectors, $\mathbf{q}_{1}^{*}, \mathbf{q}_{2}^{*}, \mathbf{q}_{3}^{*}$, and $\mathbf{q}_{4}^{*}$, the element of $\mathrm{K}$ that presents the minimum signed distance can then be determined:

$$
\min _{m, n} f\left(\mathbf{n}_{\mathrm{OP}}, \mathbf{q}_{m}^{*}, \mathbf{q}_{n}^{*}\right)
$$

with

$$
\begin{aligned}
f\left(\mathbf{n}_{\mathrm{OP}}, \mathbf{q}_{m}^{*}, \mathbf{q}_{n}^{*}\right)= & \operatorname{sign}\left(\mathbf{n}_{\mathrm{OP}} \cdot\left(\mathbf{q}_{n}^{*}-\mathbf{q}_{m}^{*}\right)\right)\left\|\mathbf{q}_{n}^{*}-\mathbf{q}_{m}^{*}\right\|_{2}= \\
& = \begin{cases}\operatorname{sign}\left(\mathbf{n}_{\mathrm{OP}} \cdot\left(\mathbf{q}_{2}^{*}-\mathbf{q}_{1}^{*}\right)\right)\left\|\mathbf{q}_{2}^{*}-\mathbf{q}_{1}^{*}\right\|_{2}, & m=1, n=2 \\
\operatorname{sign}\left(\mathbf{n}_{\mathrm{OP}} \cdot\left(\mathbf{q}_{4}^{*}-\mathbf{q}_{1}^{*}\right)\right)\left\|\mathbf{q}_{4}^{*}-\mathbf{q}_{1}^{*}\right\|_{2}, & m=1, n=4 \\
\operatorname{sign}\left(\mathbf{n}_{\mathrm{OP}} \cdot\left(\mathbf{q}_{2}^{*}-\mathbf{q}_{3}^{*}\right)\right)\left\|\mathbf{q}_{2}^{*}-\mathbf{q}_{3}^{*}\right\|_{2}, & m=3, n=2 \\
\operatorname{sign}\left(\mathbf{n}_{\mathrm{OP}} \cdot\left(\mathbf{q}_{4}^{*}-\mathbf{q}_{3}^{*}\right)\right)\left\|\mathbf{q}_{4}^{*}-\mathbf{q}_{3}^{*}\right\|_{2}, & m=3, n=4\end{cases}
\end{aligned}
$$

where $f$ is the piecewise signed Euclidean distance function, and $m \in\{1,3\}$ and $n \in\{2,4\}$ are the integer indices of the sub-vector points.

\section{RIGID SURFACE MATHEMATICAL REPRESENTATIONS AND 3-D VISUALIZATION}

The selection of a surface model to represent and visualize the body geometry is of crucial importance for contact analysis. The most important aspects to take under consideration are the geometric representativity of the surface and the analyticity of the surface functions. Preferably, the surface model must provide a compact representation (i.e., a small set of geometric parameters) that can uniquely define a surface. Quadric and superquadrics surfaces are geometric descriptions that are used to model a large variety of 3-D shapes, presenting great shape fidelity for many natural and manmade objects. In this work, the outer surface or certain regions of a body's boundary are modeled as ellipsoidal or superellipsoidal surfaces.

Here, only the implicit surface representation is considered for the contact detection methodology, whereas parametric surface functions are deduced and used solely for visualization purposes. The surfaces are defined as a polynomial function in $x, y$ and $z$ Cartesian coordinates. For this reason, such functions are 
called algebraic surfaces. Quadrics are second-degree polynomials while superquadrics are polynomials with non-negative real exponents. Some of the surface family members are (super)ellipsoids, and one and two sheet (super)hyperboloids. Associated with each surface function are geometric parameters that affect the shape, surface dimensions and overall curvature in a comprehensible manner.

\subsection{Quadric surfaces}

The implicit definition of a quadric surface in the canonical form, i.e., the spatial configuration in which the surface is centered at the origin and the main axis are aligned with the local coordinate system, is expressed as a dimensionless real valued scalar function,

$$
\mathcal{F}_{Q}(x, y, z)=a_{11} x^{2}+a_{22} y^{2}+a_{33} z^{2}+a_{1} x+a_{2} y+a_{3} z-1=0
$$

with

$$
a_{11} a_{1}=a_{22} a_{2}=a_{33} a_{3}=0
$$

where $\left\{a_{11}, a_{22}, a_{33}\right\}$ are shape coefficients and $\left\{a_{1}, a_{2}, a_{3}\right\}$ are unit or zero valued coefficients that define the quadric surface family type (as referred in Table 3). Dimension parameters along the $x, y$, and $z$ directions are given by the following formulas:

$$
a=\left|a_{11}\right|^{-1 / 2}, \quad b=\left|a_{22}\right|^{-1 / 2}, \quad c=\left|a_{33}\right|^{-1 / 2} .
$$

Table 3: Quadric family classification according to the coefficient values.

It should be noted that Eq. 25 can also be expressed in a matrix form $\mathcal{F}_{Q}(x, y, z)=\mathbf{x}^{T} \mathbf{Q} \mathbf{x}=0$, where

$$
\mathbf{x}=\left[\begin{array}{c}
x \\
y \\
z \\
1
\end{array}\right], \quad \text { and } \quad \mathbf{Q}=\left[\begin{array}{cccc}
a_{11} & 0 & 0 & \frac{1}{2} a_{1} \\
0 & a_{22} & 0 & \frac{1}{2} a_{2} \\
0 & 0 & a_{33} & \frac{1}{2} a_{3} \\
\frac{1}{2} a_{1} & \frac{1}{2} a_{2} & \frac{1}{2} a_{3} & -1
\end{array}\right]
$$

Due to the radial symmetry of the surface, a generalized angle-center parameterization can be deduced by expressing the quadric surface in spherical coordinates and by making use of the well-known quadratic formula from algebra: 


$$
\mathbf{p}_{Q}\left(\varphi, \theta ; a_{11}, a_{22}, a_{33}, a_{1}, a_{2}, a_{3}\right)=r_{Q}(\varphi, \theta)\left[\begin{array}{c}
\mathrm{s} \theta \mathrm{c} \varphi \\
\mathrm{s} \theta \mathrm{s} \varphi \\
\mathrm{c} \varphi
\end{array}\right]
$$

with

$$
r_{Q}(\varphi, \theta)=\frac{-r_{1}+\sqrt{r_{1}^{2}+4 r_{2}}}{2 r_{2}}
$$

and

$$
\left\{\begin{array} { l } 
{ r _ { 1 } = a _ { 1 } \mathrm { s } \theta \mathrm { c } \varphi + a _ { 2 } \mathrm { s } \theta s \varphi + a _ { 3 } c \theta } \\
{ r _ { 2 } = a _ { 1 1 } \mathrm { s } ^ { 2 } \theta \mathrm { c } ^ { 2 } \varphi + a _ { 2 2 } \mathrm { s } ^ { 2 } \theta \mathrm { s } ^ { 2 } \varphi + a _ { 3 3 } c ^ { 2 } \theta }
\end{array} , \quad \text { and } \quad \left\{\begin{array}{l}
\varphi \in\left[\varphi_{o}, \varphi_{i}\right] \\
\theta \in\left[\theta_{o}, \theta_{i}\right]
\end{array}\right.\right.
$$

where $\mathrm{c}=\cos (),. \mathrm{s}=\sin (),. r_{Q}$ is the radial coordinate, $\varphi$ and $\theta$ are the azimuth and zenith angular coordinates. If $r_{Q}=1$ for all the angular domain then Eq. 29 defines a unit sphere. Note that Eq. 30 is a parametric equation that is valid for all quadric surface family members listed in Table 3 . This is extremely useful in terms of computational implementation of the visualization of the contact surfaces since only one expression represents all surface members.

Given the implicit representation of the surface in the canonical form (Eq. 25), the normal vector of a quadric surface is the gradient vector of the scalar function $\mathcal{F}_{Q}$. The normal vector is therefore given by:

$$
\mathbf{n}_{Q}=\nabla \mathcal{F}_{Q}(x, y, z)=\left[\begin{array}{c}
2 a_{11} x+a_{1} \\
2 a_{22} y+a_{2} \\
2 a_{33} z+a_{3}
\end{array}\right] \text {, }
$$

The associated Jacobian matrix contribution is deduced by differential calculus as,

$$
\left(\mathbf{n}_{Q}\right)_{\mathbf{q}}=2\left[\begin{array}{ccc}
a_{11} & 0 & 0 \\
0 & a_{22} & 0 \\
0 & & a_{33}
\end{array}\right],
$$

with $\mathbf{q}=\left[\begin{array}{lll}x & y & z\end{array}\right]^{T}$.

The expressions of Eq. 25, Eq. 26, Eq. 27, Eq. 28, Eq. 29 and Eq. 30 are simple and computationally inexpensive, promptly to be assembled within the 
vector of geometric constraints and associated Jacobian matrix of the NewtonRaphson scheme (Eq. 8).

\subsection{Superquadric surfaces}

Superquadrics are a generalization of quadric surfaces with the exponent replaced by a non-negative number. Thus, relatively to quadric surfaces, the varying exponent consists of the introduction of a new degree of freedom for geometric modeling. By varying the exponent value, rounded, squared, filleted or pinched shapes can be attained. In the particular case of superellipsoids, since the proposed contact methodology refers to convex objects, the surface shape is mediated between circular and rectangular shapes (refer to Table 4).

Barr [2] presented an implicit surface representation of a superquadric which, in the canonical form, is given by the following expression:

$$
\mathcal{F}_{B}(x, y, z)=\left(a_{11} x^{\gamma_{2}}+a_{22} y^{\gamma_{2}}\right)^{\frac{\gamma_{1}}{\gamma_{2}}}+a_{33} z^{\gamma_{1}}-1=0
$$

where $\left\{a_{11}, a_{22}, a_{33}\right\}$ are shape coefficients, and $\gamma_{1}$ and $\gamma_{2}$ are the exponents. Depending on the signal value of shape coefficients, one can define the family type of the superquadric surface. If $\gamma_{1}=\gamma_{2}$ with $\gamma_{1}, \gamma_{2} \in\{1,2\}$ then Eq. 33 falls in to a quadric implicit function. Barr [2] introduced a spherical product operator through which a surface is created given two parametric curves that lay upon two orthogonal planes. In fact, it is merely a generalization of the spherical to rectangular coordinate transformation. Basically, $\gamma_{1}$ and $\gamma_{2}$ are the exponents of the orthogonal curves that lay on $y \mathrm{O} z$ and $x \mathrm{O} y$, respectively. The formulas for the dimension parameters along the $x, y$, and $z$ directions are given by:

$$
a=\left|a_{11}\right|^{-1 / \gamma_{2}}, \quad b=\left|a_{22}\right|^{-1 / \gamma_{2}}, \quad c=\left|a_{33}\right|^{-1 / \gamma_{1}} .
$$

Table 4: Superquadric family classification according to the coefficient values.

The angle-center parameterization is deduced in the same way as for the quadric surfaces. This being, the parametric superquadric representation $\mathbf{p}_{B}$ is given by: 


$$
\mathbf{p}_{B}\left(\varphi, \theta ; a_{11}, a_{22}, a_{33}, \gamma_{1}, \gamma_{2}\right)=r_{B}(\varphi, \theta)\left[\begin{array}{c}
\mathrm{s} \theta \mathrm{c} \varphi \\
\mathrm{s} \theta \mathrm{s} \varphi \\
\mathrm{c} \varphi
\end{array}\right]
$$

with

$$
r_{B}(\varphi, \theta)=\left(\mathrm{s}^{\gamma_{1}} \theta\left(a_{11} \mathrm{c}^{\gamma_{2}} \varphi+a_{22} \mathrm{~s}^{\gamma_{2}} \varphi\right)+a_{33} \mathrm{c}^{\gamma_{1}} \theta\right)^{-\frac{1}{\gamma_{1}}}, \text { and }\left\{\begin{array}{l}
\varphi \in\left[\varphi_{o}, \varphi_{i}\right] \\
\theta \in\left[\theta_{o}, \theta_{i}\right]
\end{array}\right.
$$

where $r_{B}$ is the radial coordinate, $\varphi$ and $\theta$ are the azimuth and zenith angular coordinates. Note that Eq. 36 is a parametric equation that is valid for all the mentioned superquadric family members in Table 4.

Barr's [2] implicit definition of the superquadric surface is composed by a function raised to $\gamma_{1} / \gamma_{2}$. By twice differentiating Eq. 33, the exponent $\gamma_{1} / \gamma_{2}-2$ appears in the resulting expressions. As a consequence, only values of $\gamma_{1} \geq 2 \gamma_{2}$ are permissible for the proposed contact detection method, so that non-negative exponents are preserved. This deeply hampers the desired geometric representativity and excludes the particular case of the quadric surfaces. For contact analysis with implicit surfaces, the superquadric definition proposed by Barr is quite limited, although it is entirely applicable when considering the parametric version of the geometric constraints. Therefore, an alternative quadric surface generalization is considered:

$$
\mathcal{F}_{S Q}(x, y, z)=a_{11} x^{\gamma_{1}}+a_{22} y^{\gamma_{2}}+a_{33} z^{\gamma_{3}}-1=0
$$

where $\left\{a_{11}, a_{22}, a_{33}\right\}$ are shape coefficients, and $\gamma_{1}, \gamma_{2}$, and $\gamma_{3}$ are real non-negative exponents.

In any computational implementation involving polynomials with rational exponents, awareness must be given to the numerical evaluation of superquadric equations [27]. The correct order of evaluation of these exponential terms is $\left(x^{2}\right)^{1 / \varepsilon}$, with $\gamma=2 / \mathcal{E}$, to assure that the result is not a complex number when $x<0$. In order to prevent divisions by zero, the exponent must be greater or equal to zero. The values of $\gamma_{1}, \gamma_{2}$, and $\gamma_{3}$ are bounded between 2 and infinity so that only convex shapes, with no geometric singularities, are modeled. An anglecenter parametric expression for superquadrics with $\gamma_{1}=\gamma_{2}=\gamma_{3}$ is also easily deducible. 
The normal vector to a superquadric surface is also given by the gradient vector and the Jacobian matrix is obtained in the same fashion:

$$
\begin{gathered}
\mathbf{n}_{S Q}=\nabla \mathcal{F}_{S Q}(x, y, z)=\left[\begin{array}{c}
a_{11} \gamma_{1} x\left(x^{2}\right)^{\frac{1}{\varepsilon_{1}}-1} \\
a_{22} \gamma_{2} y\left(y^{2}\right)^{\frac{1}{\varepsilon_{2}}-1} \\
a_{33} \gamma_{3} z\left(z^{2}\right)^{\frac{1}{\varepsilon_{3}}-1}
\end{array}\right], \\
\left(\mathbf{n}_{S Q}\right)_{\mathbf{q}}=\left[\begin{array}{ccc}
a_{11} \gamma_{1}\left(\gamma_{1}-1\right)\left(x^{2}\right)^{\frac{1}{\varepsilon_{1}}-1} & 0 \\
0 & a_{22} \gamma_{2}\left(\gamma_{2}-1\right)\left(y^{2}\right)^{\frac{1}{\varepsilon_{2}}-1} & 0 \\
0 & 0 & a_{33} \gamma_{3}\left(\gamma_{3}-1\right)\left(z^{2}\right)^{\frac{1}{\varepsilon_{3}}-1}
\end{array}\right],
\end{gathered}
$$

with $\mathbf{q}=\left[\begin{array}{lll}x & y & z\end{array}\right]^{T}$.

The problem in generalizing the proposed contact methodology to superquadrics is that they lead to polynomial equations with fractional exponents, which are difficult to solve numerically. Also for the case of superellipsoidal surfaces, when two local surface coordinates are close to zero, the Jacobian matrix in Eq. 39 becomes ill-conditioned, which can potentially jeopardize the nonsingularity of the Jacobian matrix of the geometric constraints given in Eq 12.

\section{PROXIMITY QUERIES}

In order to increase the efficiency of the contact detection algorithm, proximity queries are usually considered [16,18]. They consist of simple tests to ascertain if the surfaces are apart for a given instant of time.

When dealing with closed surface quadrics, such as spheres and ellipsoids, the most commonly used proximity query is the bounding sphere test. Such technique allows rapid tests for proximity contact detection queries, but is not quite precise, and is often evaluated to determine if a more detailed testing is required. A bounding sphere, as the name suggests, is a sphere that contains all the points of the ellipsoid and shares the same centroid. Basically, the test consists in evaluating the inequality between the sum of the semi-major axis of the ellipsoids and the distance between their centroids. If the distance is lesser or 
equal to the sum of the semi-major axis then a more detailed testing must be conducted. An elegant and efficient algorithm for detecting contact between two ellipsoids was presented by Choi et al. [18]. This algorithm is based on the separation condition of two ellipsoids, which is a necessary and sufficient condition, stating that the characteristic equation has positive roots if, and, only if the ellipsoids do not have common interior points [17]. The separation condition heavily depends on the matrix form of a quadric surface expressed in Eq. 28 and is valid for ellipsoids that are not contained within each other.

Unfortunately, an elegant proximity query, such as the separation condition for ellipsoids, is not, to our knowledge, available for superellipsoids.

\section{CONTACT DETECTION ALGORITHM}

Within the framework of multibody dynamics, the contact detection algorithm takes the bodies positions and orientations, calculated from the equations of motion, and returns the location of the pair of contact points together with the contact forces. The proposed algorithm can be summarized in the following steps:

(i) Establish the time interval $\left[t_{0}, t_{e n d}\right]$ for the dynamic analysis;

(ii) At time step $t_{0}$, establish the initial conditions for the position vector, $\mathbf{q}_{0}=\mathbf{q}\left(t_{0}\right)$, velocity vector, $\dot{\mathbf{q}}_{0}=\dot{\mathbf{q}}\left(t_{0}\right)$, and contact candidate pair of points, $\mathbf{q}_{0}^{G}=\mathbf{q}^{G}\left(t_{0}\right) ;$

(iii) Evaluate the proximity queries; if the surfaces are sufficiently close then go to (iv), otherwise go to (vii);

(iv) Run the Newton-Raphson method with analytical Jacobians to obtain the vector of the contact points, $\mathbf{q}^{G}$;

(v) Compute the signed distance magnitude, $d$, and check for contact; if there is contact, evaluate contact forces according to the constitutive law; if not go to step (vii);

(vi) Add the contact forces to the vector of applied forces, $\mathbf{g}$;

(vii) Solve the equations of motion deduced from the multibody dynamics formulation in order to obtain the body positions and orientations for the new time step $t+\Delta t$; 
(viii) Update the system time variable $t$ and use the vector $\mathbf{q}^{G}$ obtained in (iv) as the initial guess for the Newton-Raphson method in the next time step;

(ix) Go to step (iii) and proceed with the whole process for the new time step;

(x) Exit the main algorithm's loop when the final time step is reached.

Note that the contact detection algorithm is run $N$ times for each function evaluation, where $N$ is the number of rigid contact pairs within the multibody system. Fig. 5 shows the flowchart of the contact methodology. The numerical implementation of this methodology leads to an efficient algorithm since the information of the previous time step is used as an initial guess to find the solution of the non-linear equations and, therefore, only a few iterations are required to obtain the solution, as it will be discussed in the next section.

Figure 5: Flowchart of the proposed contact detection algorithm.

\section{RESULTS}

The results presented in this section refer to the distance computation between contact pairs of ellipsoidal (Table 5) and superellipsoidal (Table 6) surfaces in several configurations and with different geometric parameters. No proximity queries or multibody dynamics calculations are undertaken in order to evaluate, solely, the distance computation efficiency during the analysis. The contact analysis is performed for a time interval of 200 time steps and, based on numerical experiments, with a tolerance of $10^{-6}$ for the Newton-Raphson accuracy, i.e., $\left\|\Delta \mathbf{q}_{k}^{G}\right\|_{2}=\left\|\mathbf{q}_{k+1}^{G}-\mathbf{q}_{k}^{G}\right\|_{2} \leq 10^{-6}$. Prescribed motion is imposed in all cases. The initial approximation, $\mathbf{q}_{0}^{G}$, consisted of the bisection between the surface centroids. For the remaining time steps, the Newton-Raphson approximations are the resulting vector from the previous time step, i.e., $\mathbf{q}_{k}^{G}$. The contact detection code was developed in $\mathrm{MATLAB}^{\circledR}$ running on a PC with a Intel ${ }^{\circledR}$ Core 2 Duo processor $1.66 \mathrm{GHz}$ and $2 \mathrm{~GB}$ of RAM. The software code for minimum distance calculation between ellipsoids is available at [28]. 
Table 5: Contact pair tests for the calculation of the minimum distance between ellipsoid surfaces in a total of 200 time steps. Caption: Ellipsoid coefficients - $\{a, b, c\} ; \mathrm{H}-$ Householder approach; AV - Auxiliary vector approach.

Table 6: Contact pair tests for the calculation of the minimum distance between superellipsoid surfaces in a total of 200 time steps. Caption: Superellipsoid coefficients $-\left\{a, b, c, \gamma_{1}, \gamma_{2}, \gamma_{3}\right\} ; \mathrm{H}-$ Householder approach.

Figure 6: Snapshots of the minimum distance calculation for each contact pair defined in Table 5. The normal, tangent and binormal vectors are colored as red, green, and blue, respectively.

Figure 7: Snapshots of the minimum distance calculation for each contact pair defined in Table 6. The normal, tangent and binormal vectors are colored as red, green, and blue, respectively.

A high efficiency is obtained for distance calculation of a ellipsoidal surface contact pair, in which 200 time steps took a total computational time that is lesser than 1.25 seconds, and an average of 5 Newton-Raphson iterations per time step. Both approaches, the Householder transformation and the set of noncollinear auxiliary vectors, for determining tangent and binormal vectors are considered presenting no major differences regarding efficiency. Time evaluation is comparatively higher when dealing with superellipsoids due, mainly, to the greater non-linearity of the geometric constraints vector. Although all cases converged to the desired solution, in Fig. 6-2, it is shown an example of an alternative pair of points that satisfies Eq. 9.

\section{DISCUSSION}

From a mathematical point of view, ellipsoids detain a better behavior than superellipsoids since special treatment is not required for continuity singularities and the issues associated with rational exponents do not take place (divisions by zero and the appearance of complex numbers for negative domain values). On the other hand, superquadrics possess a higher geometric representativity since the varying exponents control the overall curvature of the surface, contrary to quadrics that have a constant power. Both quadric and superquadric surfaces have a small number of geometric parameters and approximate a wide class of convex objects.

Notice that the contact formulation proposed here is only applied to convex surfaces placed in a non-conformal contact situation. In fact, if one or both surfaces are concave, multiple solutions may appear. In order to apply the contact 
detection methodology to conformal situations, a set of superellipsoids can be arranged to fit the non-convex surface.

For ellipsoidal surfaces, the Jacobian matrices involved in Eq. 12 guaranteedly do not contain lines of zeros. On the other hand, for superellipsoids it is necessary to ensure that the Jacobians of the normal vector and auxiliary vectors are not ill-conditioned so that $\boldsymbol{\Phi}_{\mathbf{q}^{G}}^{G}$ does not become a singular matrix when a pair of local coordinates are close to zero. In such case, it is necessary to consider the pseudo-inverse of the geometric constraints Jacobian, although in some particular cases the Jacobian becomes singular. Another possible Jacobian singularity appears, for both ellipsoid and superellipsoid cases, when

$$
\mathbf{d}_{\mathrm{PQ}}=\mathbf{n}_{\mathrm{OP}}
$$

and

$$
\left(\mathbf{d}_{\mathrm{PQ}}\right)_{\mathbf{q}^{G}}=\left(\mathbf{n}_{\mathrm{OP}}\right)_{\mathbf{q}^{G}} \Rightarrow \mathbf{n}_{i \mathrm{P}}=\left(\mathbf{A}_{\mathrm{O} \beta} \mathbf{A}_{\beta j}\right)^{-1}\left(\mathbf{A}_{\mathrm{O} \beta} \mathbf{A}_{\beta j}-\mathbf{A}_{\mathrm{O} \alpha} \mathbf{A}_{\alpha i}\right)[\mathbf{I}: \mathbf{0}]
$$

simultaneously occur since a set of rows in Eq. 12 becomes linearly dependent. If the Jacobian matrix, which was calculated analytically, becomes singular, alternative ways to calculate the Jacobian matrix must be considered, e.g., by finite difference estimation of the Jacobian matrix or, preferably, by reformulating the vector function $\boldsymbol{\Phi}^{G}$ with other linear independent collinear or orthogonal constraints (Eq. 6a, Eq. 6b).

Additionally, in some contact pair situations, it should be noted that there may be more than one possible solutions for Eq. 9, i.e., the existence of other pairs of points that belong to the line containing the collinear normals that intersects the closed surfaces on both sides. Therefore, at each time step it is necessary to check if the obtained solution matches the minimum distance: determine the remaining 2 points by radial symmetry and the pair of points that are not associated with the maximum distance make up the desired solution (Eq. 22, Eq. 24a, Eq. 24b).

Contact calculations contribute quite significantly to the computational cost of multibody dynamics analysis. The usage of analytical Jacobians, besides guaranteeing the geometric accuracy of the result, also contributes to the computational efficiency, since no matrix estimate is required for each iteration neither does it depends on polygonal geometries nor complex non-linear optimization methods. A special remark must be drawn to the importance of the 
implicit surface representation since the methodology's success depends greatly on such a compact and well-behaved mathematical expression. All in all, the computational efficiency and robustness are the major advantages of the present model as it converges rapidly, allowing simulations to be performed interactively. Though the computational time is dependent on the initial guess (a limitation inherited from the Newton-Raphson algorithm), in all cases tested, 200 time steps were completed in less than in 1.25 seconds for ellipsoids, and 4.10 seconds for superellipsoids.

\section{CONCLUSIONS}

In this work, an accurate, efficient, and easily implementable algorithm for minimum distance computation between ellipsoidal and superellipsoidal surfaces was presented. The proposed contact methodology relies on the common normal concept and on locus constraints that are quite intuitive. Consequently, the methodology is easily formulated resorting to vector calculus and algebraic and differential geometry, providing a uniform framework for distance computation between objects described as arbitrary convex implicit surfaces (at least $\mathrm{C}^{2}$ continuous).

As major advantages towards other contact detection algorithms, the proposed contact algorithm does not resort on optimization methods or convex polyhedral geometries, making use of the potential of analytical expressions for the surface vectors and associated Jacobian matrices. The usage of analytical Jacobians guarantees geometric accuracy and contributes to the computational efficiency of the method, since no matrix estimation is required for each iteration. Mathematical artifices were introduced to compute tangent and binormal vectors for implicit surfaces given the normal vector to a surface. The speed at which distance computation is performed enables real-time simulations for a contact pair.

Despite the Newton-Raphson's local convergence behaviour and that the common normal conditions possibility on granting multiple solutions in some contact pairs, the methodology solves such issues by relying, essentially, on the radial symmetry and convexity of the (super)ellipsoidal surfaces. The main pitfall of the methodology is the possibility of the Jacobian matrices becoming singular in the abovementioned situations. 
The proposed contact methodology explores the implicit representation of superquadrics, which relies on algebraic expressions with rational exponents, contrary to the parametric counterpart which involves trigonometric functions raised to rational exponents, i.e., expressions with higher non-linearity.

Instead of presenting specific parametric expressions for each (super)quadric surface member the generic (i.e., valid for all quadric members and superellipsoid and superhyperboloid members) angle-center formulas of the parameterized surfaces were deduced and are bestowed for visualization and 3-D modeling purposes.

\section{ACKNOWLEDGEMENTS}

The authors would like to acknowledge the support given by the Fundação para a Ciência e a Tecnologia (FCT), through projects ProPaFe (PTDC/EMEPME/67687/2006) and DACHOR (MIT-Pt/BS-HHMS/0042/2008). The first author would like to thank FCT for the PhD grant SFRH/BD/47750/2008.

\section{REFERENCES}

[1] P. Nikravesh, Computer-aided analysis of mechanical systems. Prentice-Hall, Englewood Cliffs, New Jersey, 1988.

[2] A. H. Barr, Superquadrics and Angle-Preserving Transformations, IEEE Computer Graphics and Applications, 1, 1, 11-23, 1981.

[3] C. L. Li, Part Segmentation by Superquadric Fitting - A New approach Towards Automatic Design of Cooling System for Plastic Injection Mould, International Journal of Advanced Manufacturing Technology, 35:102-114, 2007.

[4] E. Bardinet, L.D. Cohen, N. Ayache, Superquadrics and Free-Form Deformations: A Global Model to Fit and Track 3D Medical Data, Lecture Notes in Computer Science, Springer, Volume 905, 319-326, 1995.

[5] R. Schwertassek, T. Klisch, Two Modelling Problems in Multibody Simulation: Flexible Bodies and Contact of Bodies, Proceedings of the NATO Advanced Study Institute on Computational Methods in Mechanisms, Invited Lectures, 1, 283-312, Varna, Bulgaria, 1997.

[6] J. Pombo, J. Ambrósio, M. Silva, A new wheel-rail contact model for railway dynamics, Vehicle System Dynamics, 45:2,165-189, 2007.

[7] B. Fallahi, S.K. Ballamudi, Computation of Common Normal between Wheel and Rail Surfaces, Proceedings of the 1st Joint International Conference on Multibody System Dynamics, May 25-27, Lappeenranta, Finland, 2010. 
[8] H. Hertz, On the contact of solids - On the Contact of Rigid Elastic Solids and on Hardness, (Translated by D. E. Jones and G. A. Schott), Miscellaneous Papers, MacMillan and Co. Ltd., London, 1896, 146-183.

[9] H. Lankarani, P. Nikravesh, Continuous Contact Force Models for Impact Analysis in Multibody Systems, Nonlinear Dynamics, 5, 193-207, 1994.

[10] K. Hunt, F. Crossley, Coefficient of Restitution Interpreted as Damping in Vibroimpact, ASME Jornal of Applied Mechanics, 42, 440-445, 1975.

[11] P. Flores, R. Leice, C. Glocker, Modeling and Analysis of Planar Rigid Multibody Systems with Translational Clearance Joints based on the Non-Smooth Dynamics Approach, Multibody System Dynamics, 23(2), 165-190, 2010.

[12] A. Donev, S. Torquato, F.H. Stillinger, Neighbor List Collision-Driven Molecular Dynamics Simulation for Nonspherical Hard Particles II: Applications to Ellipses and Ellipsoids, J. Computational Physics, 202, 765-793, 2005.

[13] X. Lin and T.-T. Ng, Contact Detection Algorithms for Three-Dimensional Ellipsoids in Discrete Element Modelling, International Journal for Numerical and Analytical Methods in Geomechanics, 19, 653-659, 1995.

[14] S. D. Kwak, L. Blankevoort, G. A. Ateshian, A Mathematical Formulation for 3D QuasiStatic Multibody Models of Diarthrodial Joints, Computer Methods in Biomechanics and Biomedical Engineering, 3, 41-64, 2000.

[15] S. Liu, C. C. L. Wang, K.-C. Hui, X. Jin, H. Zhao, Ellipsoid-tree construction for solid objects, Proceedings of the 2007 ACM symposium on Solid and physical modeling, Beijing, China, pp. 303-308, 2007.

[16] N. Chakraborty, J. Peng, S. Akella, J. Mitchell, Proximity Queries between Convex Objects: An Interior Point Approach for Implicit Surfaces, In Proceedings of the IEEE International Conference on Robotics and Automation, 1910-1916, 2006.

[17] W. Wang, J. Wang, M.-S. Kim, An Algebraic Condition for the Separation of Two Ellipsoids, Computer Aided Geometric Design, 18, 6, 531-539, 2001.

[18] Y.-K. Choi, J.-W. Chang, W. Wang, M.-S. Kim, G. Elber, Continuous Collision Detection for Ellipsoids, IEEE Transactions on Visualization and Computer Graphics, 15, 2, 311-325, 2009.

[19] C. Wellmann, C. Lillie, P. Wriggers, A Contact Detection Algorithm for Superellipsoids based on the Common-Normal Concept, Engineering Computations, 25(5), pp. 432-442, 2008.

[20] J. García de Jalón, E. Bayo, Kinematic and Dynamic Simulation of Multibody Systems: The Real Time Challenge, Springer-Verlag, New York, 1994.

[21] R.F. Portal, L.G. Sousa, J.P. Dias, Contact Detection of Convex Superquadrics Using Optimization Techniques with Graphical User Interface, In Proceedings of the 7th EUROMECH Solid Mechanics Conference, Lisboa, Portugal, 2009.

[22] M.S. Bazaraa, H.D. Sherali, C.M. Shetty, Nonlinear Programming: Theory and Algorithms, Wiley, Hoboken, New Jersey, 1993. 
[23] Grosan, A. Abraham, A New Approach for Solving Nonlinear Equations Systems, IEEE Transactions on Systems, Man, and Cybernetics-Part A: Systems and Humans, Vol. 38, Nº. 3, 689-714, 2008.

[24] K. Moustakas, D. Tzovaras, M.G. Strintzis, SQ-Map: Efficient Layered Collision Detection and Haptic Rendering, IEEE Transactions on Visualization and Computer Graphics, 80-93, 2007

[25] K. Atkinson, W. Han, Theoretical Numerical Analysis: a Functional Analysis Framework, $2^{\text {nd }}$ Edition, Springer Texts in Applied Mathematics, Springer, 2005.

[26] A. S. Householder., Unitary Triangularization of a Nonsymmetric Matrix. Journal of the Association for Computing Machinery, 4, 5, 339-342, 1958.

[27] A. Jaklic, A. Leonardis, F. Solina. Segmentation and Recovery of Superquadrics. Computational imaging and vision, Vol. 20, Kluwer, Dordrecth, 2000.

[28] MDC-ELLIPSOIDs v1.0 - Minimum distance calculation between ellipsoids described as implicit surfaces, http://web.ist.utl.pt/daniel.s.lopes/mdc-ellipsoids.html

\section{List of figure captions}

Figure 1: Multibody model of a contact pair and vector entities involved in contact analysis along with the coordinates systems (global, rigid body and surface reference frames). The detail shows the intervening vector quantities of proposed contact detection formulation.

Figure 2: Square plate rotation mechanism. The revolute joints rotate at the same angular velocity but in opposite senses.

Figure 3: "Proof by picture" of the existence of non-collinear vectors given an arbitrary vector $\mathbf{n}_{x y}$ in the 2-D plane.

Figure 4: Multiple solutions for the common normal concept. A1 - Contact pair formed by a sphere and an ellipsoid that are aligned with each other; A2 - Multiple solutions of the corresponding vector of geometric constraints $\boldsymbol{\Phi}^{G}$; B1 - Contact pair formed by a sphere and an ellipsoid that are not aligned with each other; B2 - Multiple solutions of the corresponding vector of geometric constraints $\boldsymbol{\Phi}^{G}$.

Figure 5: Flowchart of the proposed contact detection algorithm.

Figure 6: Snapshots of the minimum distance calculation for each contact pair defined in Table 5.

The normal, tangent and binormal vectors are colored as red, green, and blue, respectively.

Figure 7: Snapshots of the minimum distance calculation for each contact pair defined in Table 6.

The normal, tangent and binormal vectors are colored as red, green, and blue, respectively. 


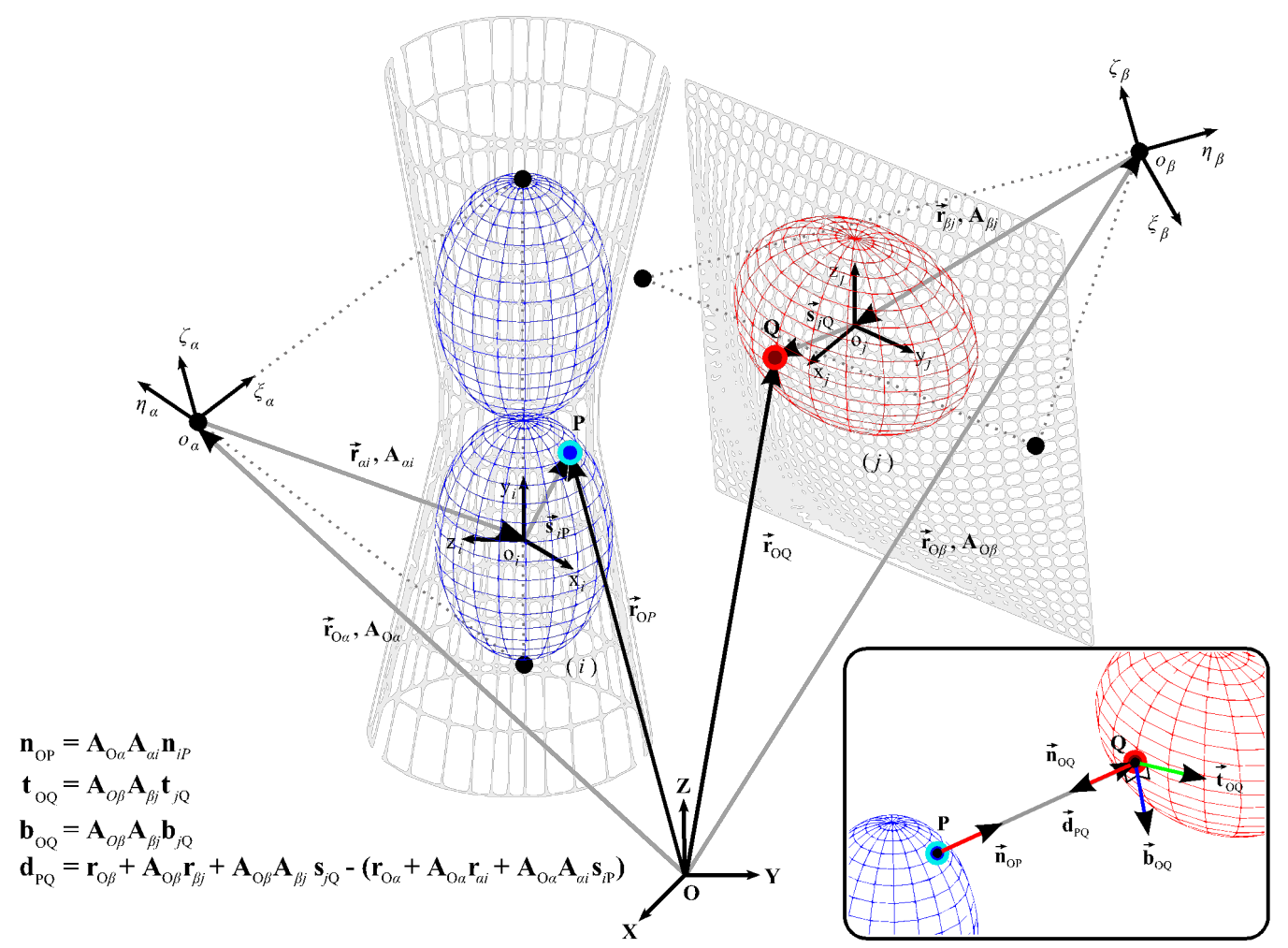

Figure 1: Multibody model of a contact pair and vector entities involved in contact analysis along with the coordinates systems (global, rigid body and surface reference frames). The detail shows the intervening vector quantities of proposed contact detection formulation. 


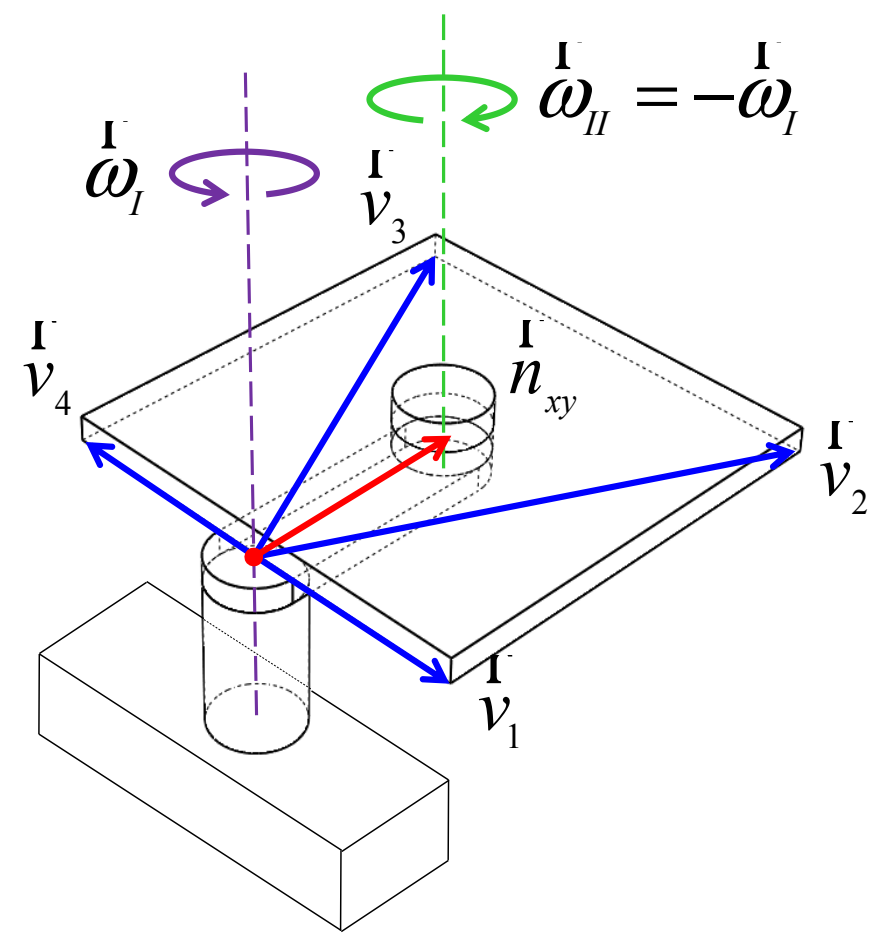

Figure 2: Square plate rotation mechanism. The revolute joints rotate at the same angular velocity but in opposite senses. 

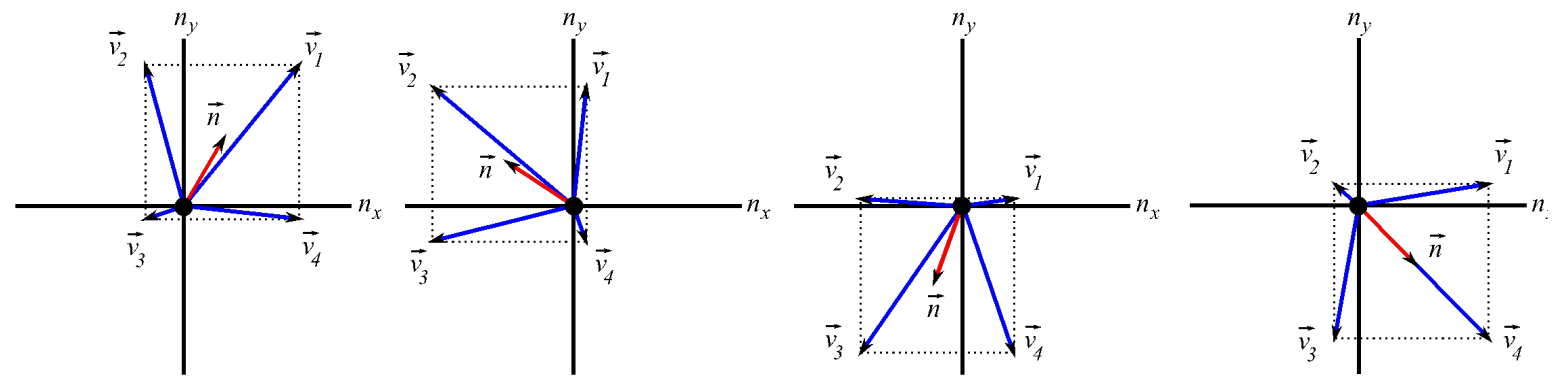

Figure 3: "Proof by picture" of the existence of non-collinear vectors given an arbitrary vector $\mathbf{n}_{x y}$ in the 2-D plane. 

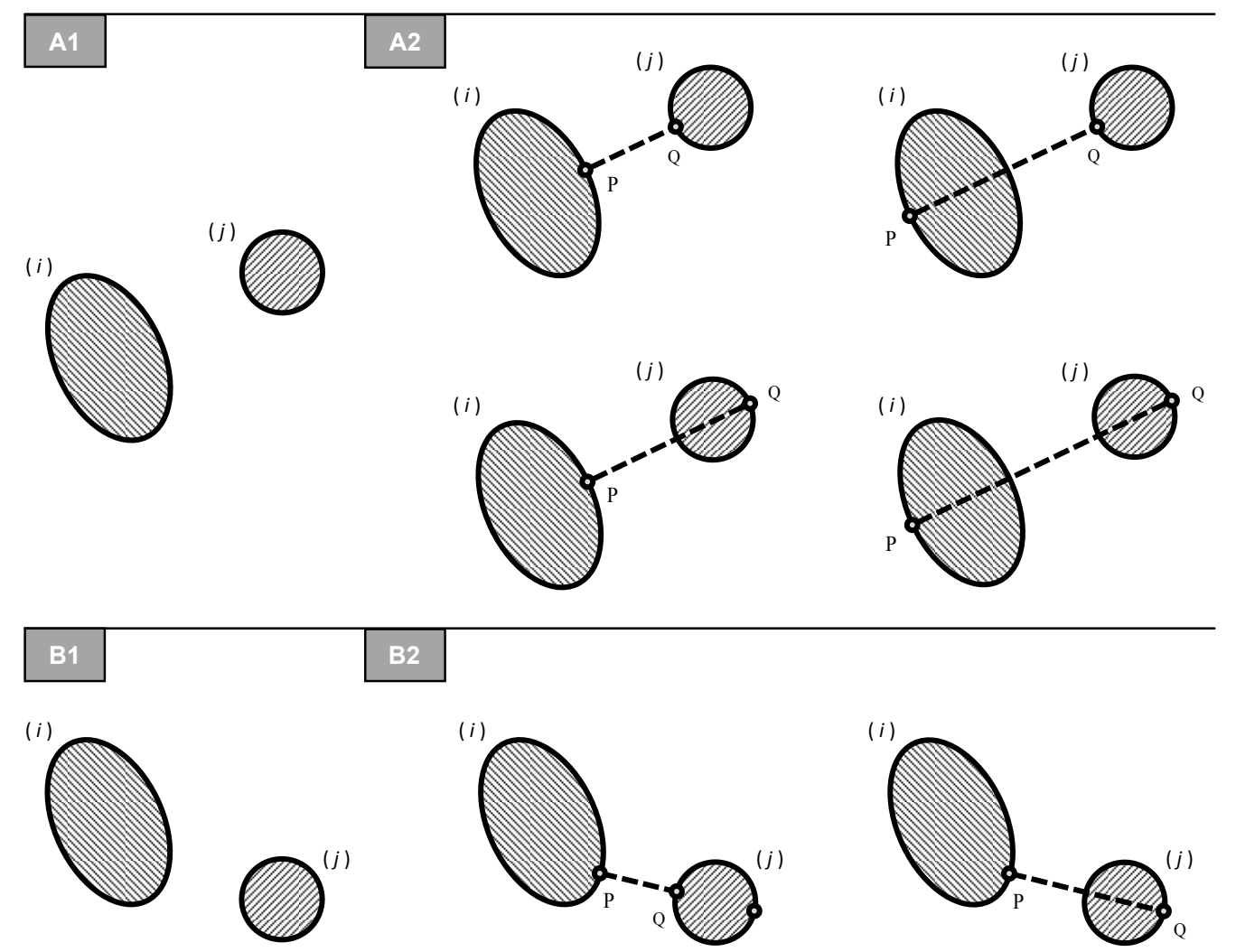

Figure 4: Multiple solutions for the common normal concept. A1 - Contact pair formed by a sphere and an ellipsoid that are aligned with each other; A2 - Multiple solutions of the corresponding vector of geometric constraints $\boldsymbol{\Phi}^{G}$; B1 - Contact pair formed by a sphere and an ellipsoid that are not aligned with each other; B2 - Multiple solutions of the corresponding vector of geometric constraints $\boldsymbol{\Phi}^{G}$. 


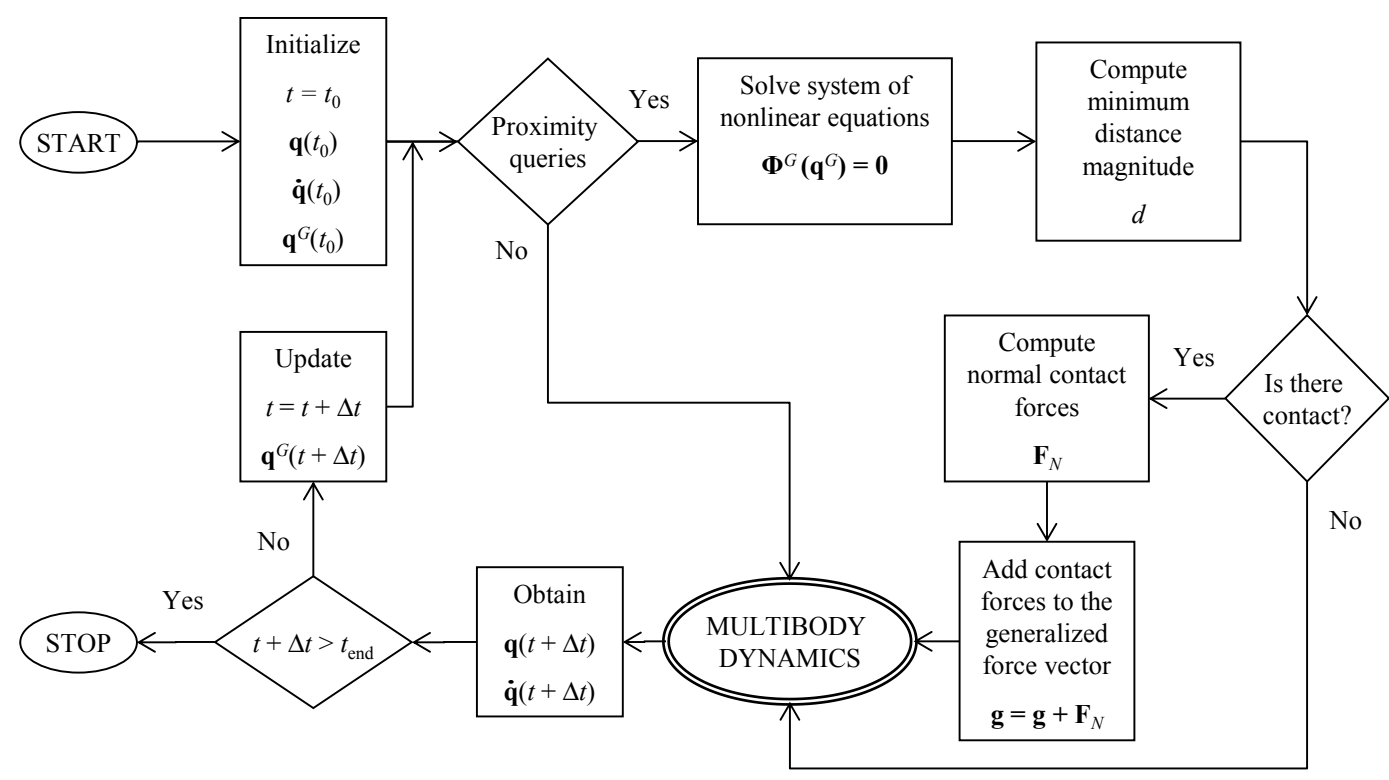

Figure 5: Flowchart of the proposed contact detection algorithm. 


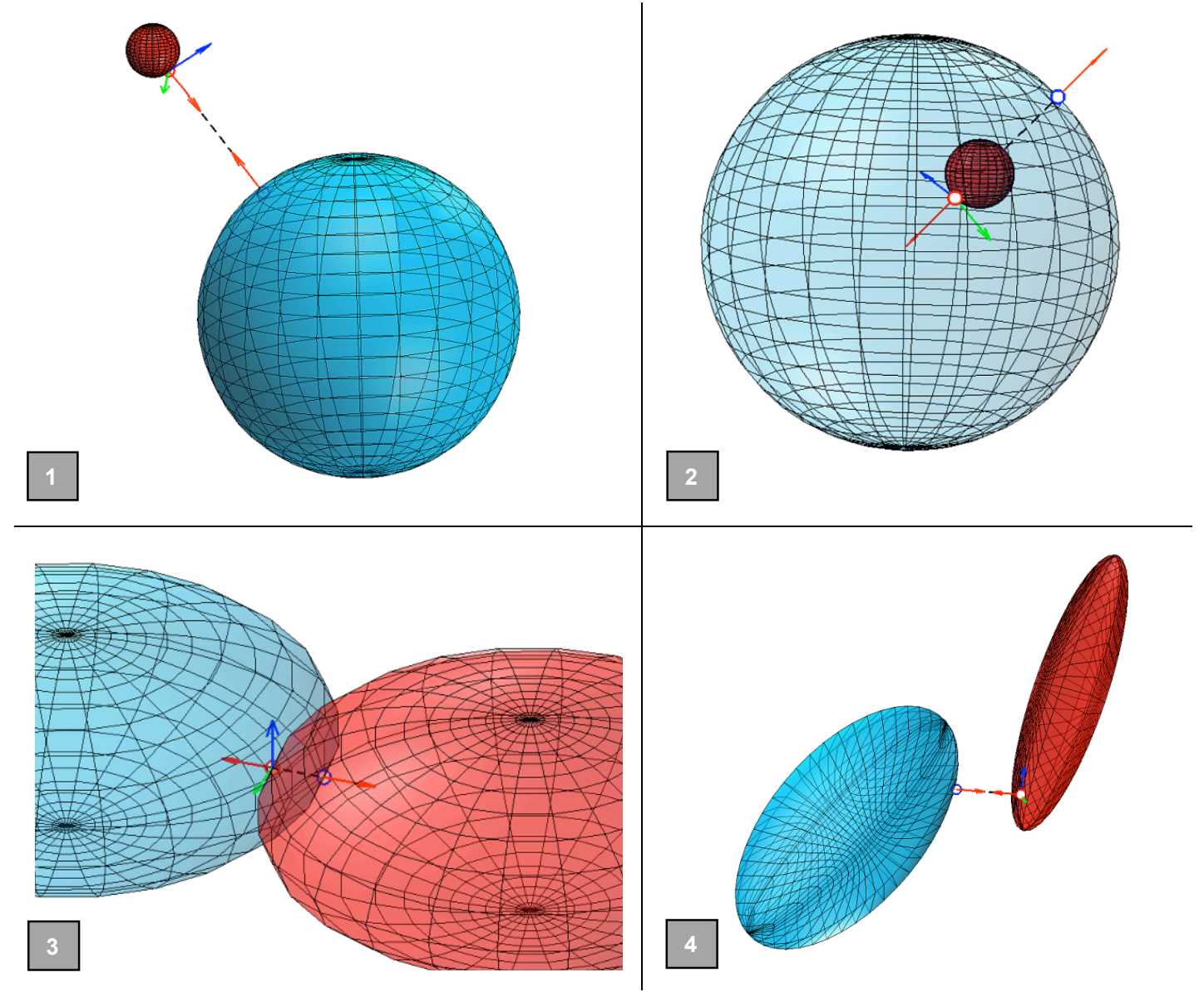

Figure 6: Snapshots of the minimum distance calculation for each contact pair defined in Table 5.

The normal, tangent and binormal vectors are colored as red, green, and blue, respectively. 

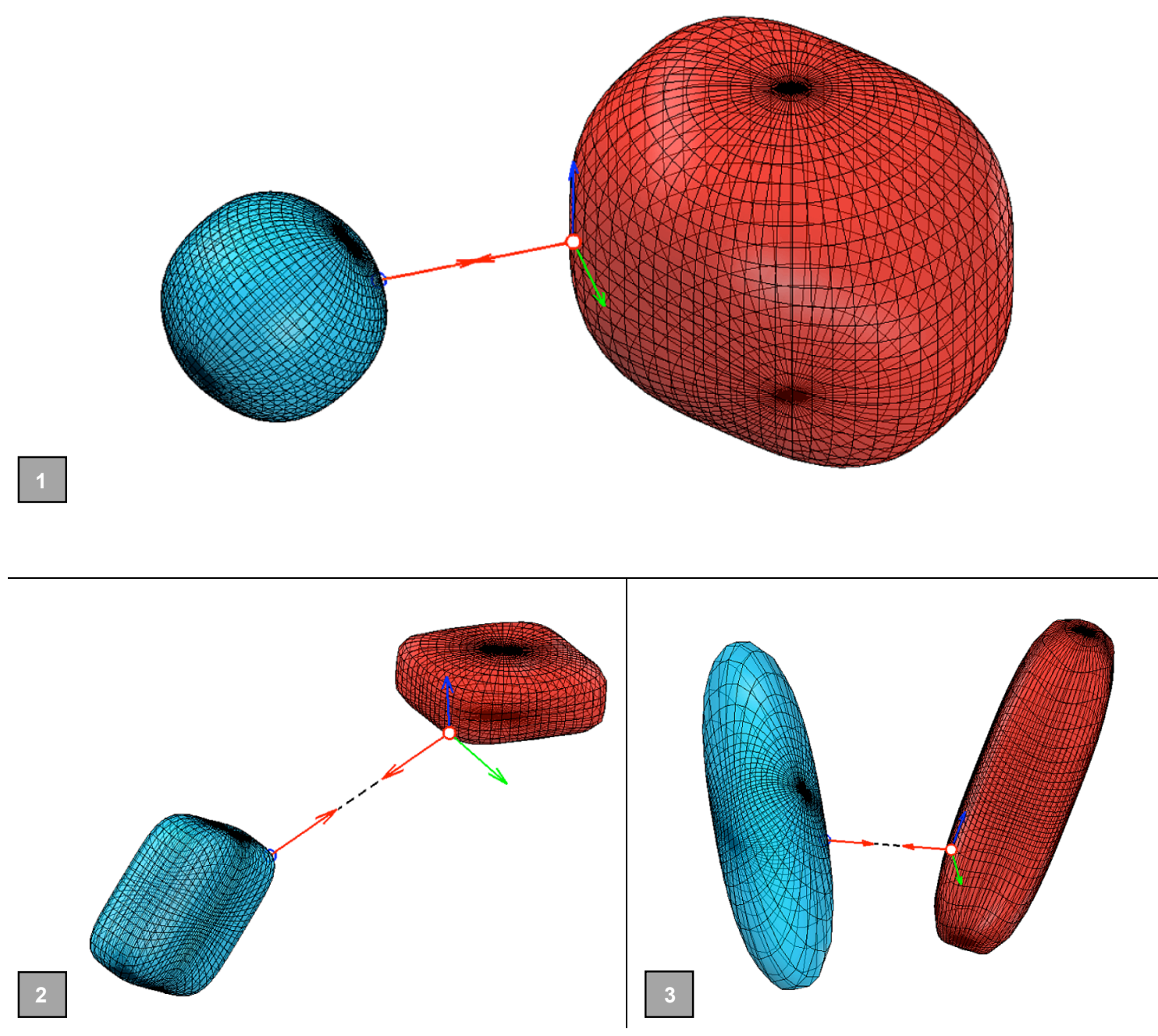

Figure 7: Snapshots of the minimum distance calculation for each contact pair defined in Table 6. The normal, tangent and binormal vectors are colored as red, green, and blue, respectively. 
Table 1: Selected pairs of non-collinear vectors according to the signs of the $n_{x}$ and $n_{y}$ coordinates.

\begin{tabular}{cccc}
\hline $\operatorname{sign}\left(n_{x}\right) \wedge \operatorname{sign}\left(n_{y}\right)$ & $\begin{array}{c}\text { Selected non- } \\
\text { collinear vector }\end{array}$ & $\begin{array}{c}\text { Sign }\left(n_{x}\right) \wedge \operatorname{sign}\left(n_{y}\right) \\
\text { collected non- }\end{array}$ \\
\hline$n_{x}>0 \wedge n_{y}>0$ & $\mathbf{v}_{2}$ or $\mathbf{v}_{4}$ & $n_{x}<0 \wedge n_{y}>0$ & $\mathbf{v}_{1}$ or $\mathbf{v}_{3}$ \\
$n_{x}=0 \wedge n_{y}>0$ & $\mathbf{v}_{2}$ or $\mathbf{v}_{4}$ & $n_{x}=0 \wedge n_{y}>0$ & $\mathbf{v}_{1}$ or $\mathbf{v}_{3}$ \\
$n_{x}>0 \wedge n_{y}=0$ & $\mathbf{v}_{2}$ or $\mathbf{v}_{4}$ & $n_{x}<0 \wedge n_{y}=0$ & $\mathbf{v}_{1}$ or $\mathbf{v}_{3}$ \\
$n_{x}<0 \wedge n_{y}<0$ & $\mathbf{v}_{2}$ or $\mathbf{v}_{4}$ & $n_{x}>0 \wedge n_{y}<0$ & $\mathbf{v}_{1}$ or $\mathbf{v}_{3}$ \\
$n_{x}=0 \wedge n_{y}<0$ & $\mathbf{v}_{2}$ or $\mathbf{v}_{4}$ & $n_{x}=0 \wedge n_{y}<0$ & $\mathbf{v}_{1}$ or $\mathbf{v}_{3}$ \\
$n_{x}<0 \wedge n_{y}=0$ & $\mathbf{v}_{2}$ or $\mathbf{v}_{4}$ & $n_{x}>0 \wedge n_{y}=0$ & $\mathbf{v}_{1}$ or $\mathbf{v}_{3}$ \\
\hline
\end{tabular}


Table 2: Contact detection situations according to the minimum distance value.

\begin{tabular}{lc}
\multicolumn{1}{c}{ Contact type } & $\begin{array}{c}\text { Minimum distance } \\
\text { No contact }\end{array}$ \\
Contact at a single point & $d=0$ \\
Contact with pseudo-penetration & $d=0$ \\
\hline
\end{tabular}


Table 3: Quadric family classification according to the coefficient values.

\begin{tabular}{lc}
\hline \multicolumn{1}{c}{ Quadric surface type } & Coefficients \\
Ellipsoid & $a_{11}>0, a_{22}>0, a_{33}>0$ \\
Hyperboloid (1 sheet) & $a_{11}>0, a_{22}>0, a_{33}<0$ \\
Hyperboloid (2 sheets) & $a_{11}<0, a_{22}<0, a_{33}>0$ \\
Paraboloid (elliptic) & $a_{11}>0, a_{22}>0, a_{33}=0, a_{3}<0$ \\
Paraboloid (hyperbolic) & $a_{11}>0, a_{22}<0, a_{33}=0, a_{3}<0$
\end{tabular}


Table 4: Superquadric family classification according to the coefficient values.

\begin{tabular}{lc}
\hline \multicolumn{1}{c}{ Superquadric surface type } & Coefficients \\
Superellipsoid & $a_{11}>0, a_{22}>0, a_{33}>0$ \\
Superhyperboloid (1 sheet) & $a_{11}>0, a_{22}>0, a_{33}<0$ \\
Superhyperboloid (2 sheets) & $a_{11}>0, a_{22}<0, a_{33}<0$
\end{tabular}


Table 5: Contact pair tests for the calculation of the minimum distance between ellipsoid surfaces in a total of 200 time steps. Caption: Ellipsoid coefficients - $\{a, b, c\} ; \mathrm{H}-$ Householder approach; AV - Auxiliary vector approach.

\begin{tabular}{|c|c|c|c|c|c|c|c|c|}
\hline Quadric Contact Pair & \multicolumn{2}{|c|}{1} & \multicolumn{2}{|c|}{2} & \multicolumn{2}{|c|}{3} & \multicolumn{2}{|c|}{4} \\
\hline Coefficients - surface $(i)$ & \multirow{2}{*}{\multicolumn{2}{|c|}{$\begin{array}{l}\{3.0,3.0,3.0\} \\
\{0.5,0.5,0.5\}\end{array}$}} & \multicolumn{2}{|c|}{$3 \cdot 0,3 \cdot 0,3 \cdot 0$} & \multicolumn{2}{|c|}{$5.0,5.0,2.0$} & \multicolumn{2}{|c|}{$1.0,5.0,5.0$} \\
\hline Coefficients - surface $(j)$ & & & \multicolumn{2}{|c|}{$0.5,0.5,0.5$} & \multicolumn{2}{|c|}{$5.0,5.0,2.0$} & \multicolumn{2}{|c|}{$5.0,1.0,5.0$} \\
\hline & \multicolumn{2}{|c|}{ - $(i)$ rotates } & \multicolumn{2}{|c|}{ - (i) rotates } & \multicolumn{2}{|c|}{ - (i) rotates } & \multicolumn{2}{|c|}{$-(i)$ rotates } \\
\hline & \multicolumn{2}{|c|}{$\mathrm{x}$-axis; } & \multicolumn{2}{|c|}{$\mathrm{x}$-axis; } & \multicolumn{2}{|c|}{ local x-axis; } & \multicolumn{2}{|l|}{$\mathrm{x}$-axis; } \\
\hline Drogoribed motion & \multicolumn{2}{|c|}{$-(j)$ rotates } & \multicolumn{2}{|c|}{ - (j) rotates } & \multicolumn{2}{|c|}{ - $(j)$ rotates } & \multicolumn{2}{|c|}{ - (j) rotates } \\
\hline Prescribea motion & \multicolumn{2}{|c|}{ along the local } & \multicolumn{2}{|c|}{ along the local } & \multicolumn{2}{|c|}{ along the } & \multicolumn{2}{|c|}{ along the local } \\
\hline & \multicolumn{2}{|c|}{$\mathrm{x}$-axis and } & \multicolumn{2}{|c|}{$\left[\begin{array}{lll}1 & 1 & 1\end{array}\right]^{\mathrm{T}}$} & \multicolumn{2}{|c|}{ local $\left[\begin{array}{lll}1 & 1 & 1\end{array}\right]^{\mathrm{T}}$} & \multicolumn{2}{|l|}{$\left[\begin{array}{lll}1 & 1 & 1\end{array}\right]^{\mathrm{T}}$} \\
\hline & \multicolumn{2}{|c|}{ orbits around } & \multicolumn{2}{|c|}{ direction. } & \multicolumn{2}{|c|}{ direction. } & direction & \\
\hline & (i). & & & & & & & \\
\hline Tangent vectors approach & $\mathbf{H}$ & AV & $\mathbf{H}$ & AV & $\mathbf{H}$ & AV & $\mathbf{H}$ & AV \\
\hline Total computational time $(s)$ & 1.18 & 1.24 & 1.23 & 0.921 & 1.15 & 1.22 & 1.20 & 1.02 \\
\hline $\begin{array}{l}\text { Total number of Newton- } \\
\text { Raphson iterations }\end{array}$ & 989 & 983 & 853 & 802 & 977 & 1005 & 956 & 1007 \\
\hline $\begin{array}{l}\text { Newton-Raphson iterations } \\
\text { per time step }\end{array}$ & $\sim 5$ & $\sim 5$ & $\sim 4$ & $\sim 4$ & $\sim 5$ & $\sim 5$ & $\sim 5$ & $\sim 5$ \\
\hline
\end{tabular}


Table 6: Contact pair tests for the calculation of the minimum distance between superellipsoid surfaces in a total of 200 time steps. Caption: Superellipsoid surface coefficients $-\left\{a, b, c, \gamma_{1}, \gamma_{2}, \gamma_{3}\right\}$; $\mathrm{H}$ - Householder approach.

\begin{tabular}{|c|c|c|c|}
\hline Superquadric Contact Pair & 1 & 2 & 3 \\
\hline Coefficients - surface $(i)$ & $\begin{array}{c}\{1.0,1.0,1.0, \ldots \\
2.4,2.4,2.4\}\end{array}$ & $\begin{array}{c}\{0.4,1.1,1.1, \ldots \\
3.2,3.2,3.2\}\end{array}$ & $\begin{array}{c}\{4.1,1.1,1.1, \ldots \\
2.5,2.5,2.5\}\end{array}$ \\
\hline Coefficients - surface $(j)$ & $\begin{array}{c}\{1.5,2.1,1.9, \ldots \\
3.0,3.0,3.0\}\end{array}$ & $\begin{array}{c}\{1.0,1.0,0.4, \ldots \\
3.2,3.2,3.2\}\end{array}$ & $\begin{array}{c}\{1.0,1.0,4.0, \ldots \\
3.2,3.2,3.2\}\end{array}$ \\
\hline Prescribed motion & $\begin{array}{l}-(i) \text { rotates along } \\
\text { the local x-axis; } \\
\text { - }(j) \text { rotates along } \\
\text { the local }\left[\begin{array}{lll}1 & 1 & 1\end{array}\right]^{\mathrm{T}} \\
\text { direction. }\end{array}$ & $\begin{array}{l}\text { - }(i) \text { rotates along } \\
\text { the local x-axis; } \\
\text { - }(j) \text { rotates along } \\
\text { the local z-axis. }\end{array}$ & $\begin{array}{l}\text { - }(i) \text { rotates along } \\
\text { the local x-axis; } \\
\text { - }(j) \text { rotates along } \\
\text { the local z-axis. }\end{array}$ \\
\hline Tangent vectors approach & $\mathbf{H}$ & $\mathbf{H}$ & $\mathbf{H}$ \\
\hline Total computational time $(s)$ & 1.24 & 1.97 & 1.39 \\
\hline $\begin{array}{l}\text { Total number of Newton- } \\
\text { Raphson iterations }\end{array}$ & 1085 & 1632 & 1074 \\
\hline $\begin{array}{l}\text { Newton-Raphson iterations per } \\
\text { time step }\end{array}$ & $\sim 5$ & $\sim 8$ & $\sim 5$ \\
\hline
\end{tabular}

